\title{
EFFECTIVE INTERACTIONS ARE EFFECTIVE INTERACTIONS
}

\author{
BARRY R. HOLSTEIN \\ Department of Physics, University of Massachusetts \\ Amherst, MA 01003, USA \\ E-mail: holstein@physics.umass.edu
}

\begin{abstract}
The subject of effective interactions is introduced and applications in both quantum mechanics and quantum field theory are presented. In particular the use of chiral perturbation theory as an effective low energy description of QCD is developed and it is argued that such methods really are effective in both the meson and baryonic sectors.
\end{abstract}

\section{Introduction}

The subject of effective interactions has become widespread in physics and the goal of these lectures is to try to present the subject in a somewhat didactic fashion within the context of low energy quantum chromodynamics-QCD. I have chosen my title - "Effective Interactions are Effective Interactions" - in order to emphasize the feature that the word "effective" has two quite different meanings in English and that both are relevant to our discussion. One meaning emphasizes that the interaction which is being used is not the real thing but rather tries to mock up the manifestations of the true underlying dynamics (in our case QCD). The other meaning is that the interaction is effective - it works! - and I will spend the rest of my time trying to convince you that both meanings are valid.

\section{What is an Effective Interaction?}

The power of effective field theory is associated with the feature that there exist many situations in physics involving two scales, one heavy and one light. Then, provided one is working at energies small compared to the heavy scale, it is possible to fully describe the interactions in terms of an "effective" picture, which is written only in terms of the light degrees of freedom, but which fully includes the influence of the heavy mass scale through its virtual effects. A number of very nice review articles on effective field theory can be found in ref. 1 .

In quantum field theory it is easy to represent what is going on. If $\phi, \Phi$ represents the light, heavy field respectively, then the functional integral which 
characterizes the full quantized theory is given by

$$
W=\int[d \phi][d \Phi] \exp i \int d^{4} x \mathcal{L}(\phi, \Phi)
$$

Now suppose we integrate out the heavy degrees of freedom-what is left is a functional integral in terms of a generally non-local "effective" interaction which characterizes the theory in terms of only the light degree of freedom $\phi$

$$
W=N \int[d \phi] \exp i \int d^{4} x \mathcal{L}_{e f f}(\phi)
$$

Now while this procedure is formally correct, the physics of what is going on] is also somewhat nonintuitive, at least to me. So before proceeding to the complex subject of application to QCD, it is useful to study this idea in the simpler context of ordinary quantum mechanics, in order to get familiar with the idea. Specifically, we examine the question of why the sky is blue, whose answer can be found in an analysis of the scattering of photons from the sun by atoms in the atmosphere - Compton scatteringd. First we examine the problem using traditional quantum mechanics and, for simplicity, consider elastic (Rayleigh) scattering from single-electron (hydrogen) atoms. The appropriate Hamiltonian is then

$$
H=\frac{(\vec{p}-e \vec{A})^{2}}{2 m}+e \phi
$$

and the leading - $\mathcal{O}\left(e^{2}\right)$-amplitude for Compton scattering is found from calculating the diagrams shown in Figure 1, yielding the familiar KramersHeisenberg form

$$
\begin{aligned}
\text { Amp } & =-\frac{e^{2} / m}{\sqrt{2 \omega_{i} 2 \omega_{f}}}\left[\hat{\epsilon}_{i} \cdot \hat{\epsilon}_{f}^{*}+\frac{1}{m} \sum_{n}\left(\frac{\hat{\epsilon}_{f}^{*} \cdot<0\left|\vec{p} e^{-i \vec{q}_{f} \cdot \vec{r}}\right| n>\hat{\epsilon}_{i} \cdot<n\left|\vec{p} e^{i \vec{q}_{i} \cdot \vec{r}}\right| 0>}{\omega_{i}+E_{0}-E_{n}}\right.\right. \\
& \left.\left.+\frac{\hat{\epsilon}_{i} \cdot<0\left|\vec{p} e^{i \vec{q}_{i} \cdot \vec{r}}\right| n>\hat{\epsilon}_{f}^{*} \cdot<n\left|\vec{p} e^{-i \vec{q}_{f} \cdot \vec{r}}\right| 0>}{E_{0}-\omega_{f}-E_{n}}\right)\right]
\end{aligned}
$$

where $\mid 0>$ represents the hydrogen ground state having binding energy $E_{0}$.

Here the leading component is the familiar $\omega$-independent Thomson amplitude and would appear naively to lead to an energy-independent cross-section. However, this is not the case. Indeed, by expanding in $\omega$ and using a few quantum mechanical identities one can show that, provided that the energy of the photon is much smaller than a typical excitation energy - as is the case for optical photons, the cross section can be written as

$$
\frac{d \sigma}{d \Omega}=\lambda^{2} \omega^{4}\left|\hat{\epsilon}_{f}^{*} \cdot \hat{\epsilon}_{i}\right|^{2}\left(1+\mathcal{O}\left(\frac{\omega^{2}}{(\Delta E)^{2}}\right)\right)
$$




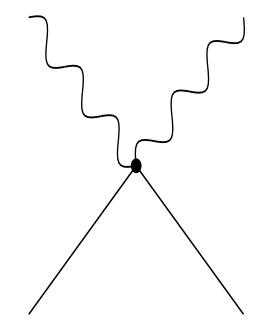

(a)

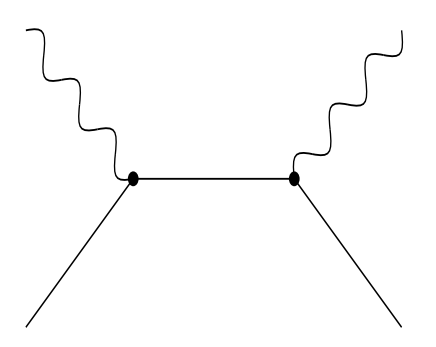

(b)

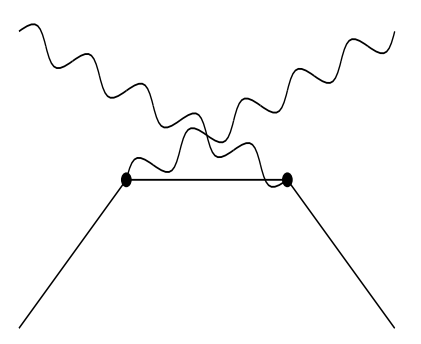

(c)

Figure 1: Feynman diagrams for nonrelativistic photonl-atom scattering.

where

$$
\lambda=\alpha_{e m} \sum \frac{2\left|z_{n 0}\right|^{2}}{E_{n}-E_{0}}
$$

is the atomic electric polarizability, $\alpha_{e m}=e^{2} / 4 \pi$ is the fine structure constant, and $\Delta E \sim m \alpha_{e m}^{2}$ is a typical hydrogen excitation energy. We note that $\alpha_{e m} \lambda \sim$ $a_{0}^{2} \times \frac{\alpha_{e m}}{\Delta E} \sim a_{0}^{3}$ is of order the atomic volume, as will be exploited below, and that the cross section itself has the characteristic $\omega^{4}$ dependence which leads to the blueness of the sky - blue light scatters much more strongly than red.

Now while the above derivation is certainly correct, it requires somewhat detailed and lengthy quantum mechanical manipulations which obscure the relatively simple physics involved. One can avoid these problems by the use of effective field theory methods outlined above. The key point is that of scale. Since the incident photons have wavelengths $\lambda \sim 5000 \mathrm{~A}$ much larger than the $\sim 1 \mathrm{~A}$ atomic size, then at leading order the photon is insensitive to the presence of the atom, since the latter is electrically neutral. If $\chi$ represents the wavefunction of the atom then the effective leading order Hamiltonian is simply that for the hydrogen atom

$$
H_{e f f}^{(0)}=\chi^{*}\left(\frac{\vec{p}^{2}}{2 m}+e \phi\right) \chi
$$

and there is no interaction with the field. In higher orders, there can exist such atom-field interactions and this is where the effective Hamiltonian comes in to play. In order to construct the effective interaction, we demand certain general principles - the Hamiltonian must satisfy fundamental symmetry requirements. In particular $H_{\text {eff }}$ must be gauge invariant, must be a scalar under rotations, and must be even under both parity and time reversal transformations. Also, 
since we are dealing with Compton scattering, $H_{\text {eff }}$ must be quadratic in the vector potential. Actually, from the requirement of gauge invariance it is clear that the effective interaction should involve only the electric and magnetic fields, rather than the vector potential itself-

$$
\vec{E}=-\vec{\nabla} \phi-\frac{\partial}{\partial t} \vec{A}, \quad \vec{B}=\vec{\nabla} \times \vec{A}
$$

since these are invariant under a gauge transformation

$$
\phi \rightarrow \phi+\frac{\partial}{\partial t} \Lambda, \quad \vec{A} \rightarrow \vec{A}-\vec{\nabla} \Lambda
$$

while the vector and/or scalar potentials are not. The lowest order interaction then can involve only the rotational invariants $\vec{E}^{2}, \vec{B}^{2}$ and $\vec{E} \cdot \vec{B}$. However, under spatial inversion $-\vec{r} \rightarrow-\vec{r}$ - electric and magnetic fields behave oppositely- $\vec{E} \rightarrow-\vec{E}$ while $\vec{B} \rightarrow \vec{B}$ - so that parity invariance rules out any dependence on $\vec{E} \cdot \vec{B}$. Likewise under time reversal invariance $\vec{E} \rightarrow \vec{E}, \vec{B} \rightarrow-\vec{B}$ so such a term is also T-odd. The simplest such effective Hamiltonian must then have the form

$$
H_{\text {eff }}^{(1)}=\chi^{*} \chi\left[-\frac{1}{2} c_{E} \vec{E}^{2}-\frac{1}{2} c_{B} \vec{B}^{2}\right]
$$

(Forms involving time or spatial derivatives are much smaller.) We know from electrodynamics that $\frac{1}{2}\left(\vec{E}^{2}+\vec{B}^{2}\right)$ represents the field energy per unit volume, so by dimensional arguments, in order to represent an energy in Eq. 10, $c_{E}, c_{B}$ must have dimensions of volume. Also, since the photon has such a long wavelength, there is no penetration of the atom, so only classical scattering is allowed. The relevant scale must then be atomic size so that we can write

$$
c_{E}=k_{E} a_{0}^{3}, \quad c_{B}=k_{B} a_{0}^{3}
$$

where we expect $k_{E}, k_{B} \sim \mathcal{O}(1)$. Finally, since for photons with polarization $\hat{\epsilon}$ and four-momentum $q_{\mu}$ we identify $\vec{A}(x)=\hat{\epsilon} \exp (-i q \cdot x)$ then from Eq. 8 , $|\vec{E}| \sim \omega,|\vec{B}| \sim|\vec{k}|=\omega$ and

$$
\frac{d \sigma}{d \Omega} \propto|<f| H_{e f f}|i>|^{2} \sim \omega^{4} a_{0}^{6}
$$

as found in the previous section via detailed calculation. This is a nice example of the power of simple effective field theory arguments. 


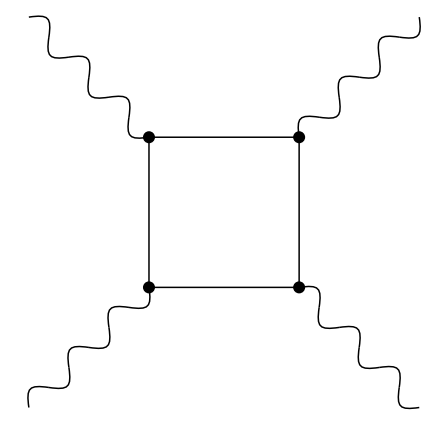

Figure 2: Charged particle box diagram contributing to photon-photon scattering.

\subsection{Euler-Heisenberg Lagrangian}

Now consider a second example - photon-photon scattering. In this case, since the photon couples to charge but is itself uncharged, there exists no lowest order interaction. However, the $\gamma \gamma \rightarrow \gamma \gamma$ process can proceed via the charged particle box diagram shown in Figure 2 .

Direct evaluation of such a diagram is straightforward but exceedingly tedious. Nevertheless, the form of the result in the case of scattering of photons with energy much smaller than the mass of the charged particles is clear from the feature that it must be representable in terms of a simple local interaction. Since photon-photon scattering is involved, the effective Lagrangian must be quartic in the vector potential and, since it must be gauge invariant, only the field tensors $F_{\mu \nu}=\partial_{\mu} A_{\nu}-\partial_{\nu} A_{\mu}$, its dual $\tilde{F}_{\mu \nu}=\frac{1}{2} \epsilon_{\mu \nu \alpha \beta} F^{\alpha \beta}$ and their derivatives can be utilized. Finally, the effective Lagrangian must be a Lorentz scalar and be parity even-i.e., to lowest order it must have the form

$$
\mathcal{L}_{\text {eff }}=\left(\frac{\alpha}{m^{2}}\right)^{2}\left[c_{1}\left(F_{\mu \nu} F^{\mu \nu}\right)^{2}+c_{2}\left(F_{\mu \nu} \tilde{F}^{\mu \nu}\right)^{2}\right]
$$

where we expect $c_{1}, c_{2} \sim \mathcal{O}(1)$. In fact explicit evaluation yields

$$
\begin{array}{ll}
c_{1}=\frac{7}{1440}, \quad c_{2}=\frac{1}{1440} & S=0 \\
c_{1}=\frac{1}{90}, & c_{2}=\frac{7}{360} \quad S=1 / 2
\end{array}
$$

which are the well-known Euler-Heisenberg results 6 Diagramatically this form corresponds to reduction of the box graph to a point interaction in accord with our discussion above. 


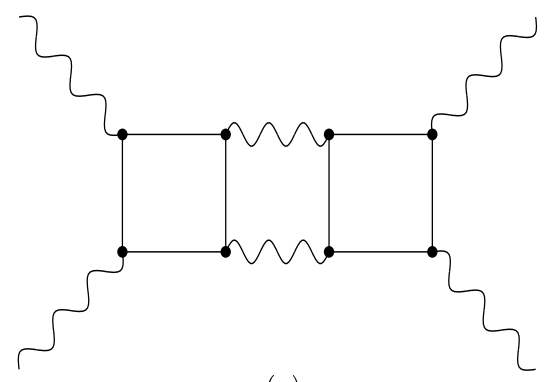

(a)

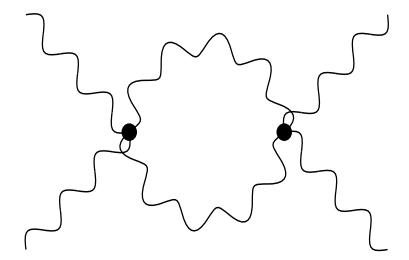

(b)

Figure 3: A two-box QED diagram and its effective action analog.

An interesting and instructive situation arises associated with the diagram shown in figure 3a, wherein two box diagrams are encoded In this case, by taking a cut across the two photons in the middle of the graph unitarity requires a non-zero imaginary component - the diagram is complex! However, such a complex number cannot be used as the coefficient of a local interaction term, as it would violate hermiticity. The resolution of this apparent paradox is that our Euler-Heisenberg contact interaction itself should be iterated in order to give the correct representation of the low energy field theory - cf. Figure 3b. The (complex) low energy - long distance component of this graph is guaranteed to reproduce the (complex) low energy piece of the full graph Figure 3a. However, an additional problem appears to surface when the effective field theory loop in figure $3 \mathrm{~b}$ is actually calculated, since it will have the basic form

$$
J(q) \sim \int \frac{d^{4} s}{(2 \pi)^{4}} \frac{\alpha^{2}}{m^{4}} \frac{q^{2} s \cdot(s+q) q^{2} s \cdot(s+q)}{s^{2}(s+q)^{2}},
$$

where $q$ represents an external momentum. Hence $J(q)$ is divergent-in dimensional regularization it will have the form

$$
J(q) \sim \frac{\alpha^{4} q^{8}}{m^{8}} \times\left(\frac{1}{d-4}-\gamma+\ldots\right)
$$

- while the QED diagram which it is supposed to represent is finite.

The resolution of this apparent paradox is that only the low energy component of the loop integration is guaranteed to match in the full and effective loop diagrams. This assures that the long distance pieces, including the imaginary part discussed above, will agree. As for the short distance piece, we must requere that the full effective theory contain a complete set of possible local 
interactions - -it must be of the form

$$
\mathcal{L}_{\text {eff }}=-\frac{1}{4} F^{2}+\frac{\alpha^{2}}{m^{4}}\left(F^{2}\right)^{2}+\frac{d_{1}}{m^{6}} F^{2} \square F^{2}+\frac{d_{2}}{m^{8}} F^{2} \square^{2} F^{2}+\ldots
$$

Here the coefficient of the term in $\left(F^{2}\right)^{2}$ yields simply the Euler-Heisenberg Lagrangian, while the remaining terms $d_{1}, d_{2}$,etc. are yet to be determined and are found by matching to the values calculated in the full theory. The solution to our dilemma is clear then-the divergence in Eq. 16 is simply combined with the phenomenological coefficient $d_{2}$ before matching to the full theory.

\subsection{Superconductivity}

Our last example before undertaking our goal of developing an effective field theory for low energy QCD is that of superconductivity. In this case the underlying dynamics is associated with that of interactions of electrons with the lattice and is described in terms of electron-phonon interactions. Equivalently one can integrate out the lattice and write things in terms of an effective interaction involving only electron degrees of freedom. In this case it was shown in the 1950's by Cooper that there develops an attractive interaction between electron pairs near the Fermi surface having opposite spins and momenta. One can think of this qualitatively in terms of the presence of the first electron attracting the lattice atom, which via screening then makes it easier for the second electron to be present. In any case one has then the effective interaction

$$
\mathcal{L}_{e f f}=\sum_{\vec{k}, s} \psi_{s}^{\dagger}(\vec{k})\left(i \frac{\partial}{\partial t}-\frac{\vec{k}^{2}}{2 m}-\mu\right) \psi_{s}(\vec{k})+g \sum_{\vec{k}, \vec{k}^{\prime}} \psi_{\uparrow}^{\dagger}(\vec{k}) \psi_{\downarrow}^{\dagger}(-\vec{k}) \psi_{\downarrow}\left(-\vec{k}^{\prime}\right) \psi_{\uparrow}\left(\vec{k}^{\prime}\right)
$$

where $\mu$ is the chemical potential. The usual (BCS) approach at this point is to solve this system using many body techniques, which leads to a ground state involving a lowered energy due to condensation of these paired electron states and which is separated by an energy gap $E_{g}$ between itself and its lowest excited state. It is this "bosonization" property and energy gap which then leads to superconductivity.

Unfortunately, this procedure is somewhat complicated and the physics is not so easy to pick out from the detailed dynamical calculations which are involved. One can ameliorate this problem, however, by writing things in terms of a two-electron state $\phi$ and integrating out the electron states to yield an effective interaction. Roughly we are doing can be seen via

$$
W=\int[d \psi]\left[d \psi^{\dagger}\right][d \phi] \delta[\phi-\psi \psi] \exp i \int d^{3} x \mathcal{L}\left(\psi^{\dagger}, \phi\right)
$$




$$
=\int[d \phi] \exp i \int d^{3} \mathcal{L}_{\text {eff }}[\phi]
$$

and diagrammatically the process can be represented as in Figure ???, where one uses imaginary time techniques in order to produce results at fixed temperature. The result of this process is the effective Hamiltonian

$$
\mathcal{H}_{e f f}=-a(T) \phi^{\dagger} \frac{D^{2}}{2 m^{*}} \phi+b(T) \phi^{\dagger} \phi+c(T)\left(\phi^{\dagger} \phi\right)^{2}
$$

where here $i D=-i \vec{\nabla}-e^{*} \vec{A}$ is the covariant derivative, $m^{*}=2 m, e^{*}=2 e$ are the effective mass, charge of the electron pairs, and $a(T), b(T), c(T)$ are calculable functions of the temperature. However, while $a(T), c(T)$ are positive and monotonic, $b(T)$ has the form $\ln T / T_{c}$ where $T_{c}$ is a critical temperature defined in terms of the coupling constant $g$. Then above this temperature, the effective potential is quartic in shape with a minimum at $\phi=0$. However, for $T<T_{c}$ the shape of the potential becomes as seen in Figure and a minimum develops at a nonzero value of $\phi$, corresponding to the existence of a superconducting state. Also, we observe that Eq. 20 has the form of the well known Landau-Ginzburg Hamiltonian, in which the physics of superconductivity is easily seen.

\subsection{QCD at Last}

After this introduction we finally turn to our goal of QCD. I was a student in the 1960's and at that time our holy grail was to attempt to find a renormalizable field theory which describes all particle interactions with the same sort of success as quantum electrodynamics (QED). In 1967 we went part of the way with development of the Weinberg-Salam theory, which incorporated the weak interaction as a sibling to the electromagnetic. Because the interaction was weak it could be treated via the same perturbative techniques as could its electromagnetic kin and what has resulted is an extremely successful description of all weak and electromagnetic processes.

For the strong interactions a renormalizable picture has also been developedquantum chromodynamics or QCD. The theory is, of course, deceptively simple 
on the surface. Indeed the form of the Lagrangian $]^{a}$

$$
\mathcal{L}_{\mathrm{QCD}}=\bar{q}(i \not D-m) q-\frac{1}{2} \operatorname{tr} G_{\mu \nu} G^{\mu \nu} .
$$

is elegant, and the theory is renormalizable. So why are we not satisfied? While at the very largest energies, asymptotic freedom allows the use of perturbative techniques, for those who are interested in making contact with low energy experimental findings there exist at least three fundamental difficulties:

i) QCD is written in terms of the "wrong" degrees of freedom-quarks and gluons - while low energy experiments are performed with hadronic bound states;

ii) the theory is non-linear due to gluon self interaction;

iii) the theory is one of strong coupling $-g^{2} / 4 \pi \sim 1-$ so that perturbative methods are not practical.

So what can be done. Using our example of superconductivity we have seen how the complicated BCS interaction in terms of spin-correlated electrons can be replaced by a relatively simple effective (Landau-Ginzburg) interaction in terms of a bound electron pair wavefunction $\phi$, wherein the physics is clarified. In the same way we shall try to do the same thing for QCD, replacing the relatively complicated interaction in terms of quark and gluon degrees of freedom by a simple form in terms of its $\bar{q} q$ and $q q q$ bound states - i.e. mesons and baryons. We shall accomplish this by exploting the chiral symmetry of the QCD interaction.

\section{$3 \quad$ Symmetry and Symmetry Breaking}

\subsection{Symmetry}

The best definition of symmetry for our purposes is probably that due to the mathematician Herman Weyl who said that a system is symmetric when

${ }^{a}$ Here the covariant derivative is

$$
i D_{\mu}=i \partial_{\mu}-g A_{\mu}^{a} \frac{\lambda^{a}}{2}
$$

where $\lambda^{a}$ (with $a=1, \ldots, 8$ ) are the $\mathrm{SU}(3)$ Gell-Mann matrices, operating in color space, and the color-field tensor is defined by

$$
G_{\mu \nu}=\partial_{\mu} A_{\nu}-\partial_{\nu} A_{\mu}-g\left[A_{\mu}, A_{\nu}\right],
$$


one can do something to it and, after making this change, the system looks the same as it did before 3 The importance of symmetry in physics is due to an important result-Noether's theorem - which connects each symmetry of a system with a corresponding conserved current and conservation law $\mathrm{g}$ Familiar examples include:

- $\mathcal{L}$ invariant under translation $\rightarrow$ momentum conservation

$-\mathcal{L}$ invariant under time translation $\rightarrow$ energy conservation

- $\mathcal{L}$ invariant under rotation $\rightarrow$ angular momentum conservation

and associated with each such invariance there is in general a related current $j_{\mu}$ which is conserved-i.e. $\partial^{\mu} j_{\mu}=0$. This guarantees that the associated charge will be time-independent, since

$$
\frac{d Q}{d t}=\int d^{3} x \frac{\partial j_{0}}{\partial t}=-\int d^{3} x \vec{\nabla} \cdot \vec{j}=\int_{\text {surf }} d \vec{S} \cdot \vec{j}=0
$$

where we have used Gauss' theorem and the assumption that any fields are local. Given a specific field theory, we can identify the Noether currents via standard techniques. Suppose that the Lagrangian is invariant under the transformation $\phi \longrightarrow \phi+\varepsilon f(\phi)$-i.e.

$$
\begin{aligned}
0 & =\mathcal{L}\left(\phi+\varepsilon f, \partial_{\mu} \phi+\varepsilon \partial_{\mu} f\right)-\mathcal{L}\left(\phi, \partial_{\mu} \phi\right) \\
& =\varepsilon f \frac{\delta \mathcal{L}}{\delta \phi}+\varepsilon \partial_{\mu} f \frac{\delta \mathcal{L}}{\delta\left(\partial_{\mu} \phi\right)}=\varepsilon \partial_{\mu}\left(f \frac{\delta \mathcal{L}}{\delta\left(\partial_{\mu} \phi\right)}\right) .
\end{aligned}
$$

so that we can identify the associated conserved current as

$$
j^{\mu}=f \frac{\delta \mathcal{L}}{\delta\left(\partial_{\mu} \phi\right)},
$$

${ }^{b}$ Note: This is often written in an alternative fashion by introducing a local transformation $\varepsilon=\varepsilon(x)$, so that the Lagrangian transforms as

$$
\mathcal{L}\left(\phi, \partial_{\mu} \phi\right) \rightarrow \mathcal{L}\left(\phi+\varepsilon f, \partial_{\mu} \phi+\varepsilon \partial_{\mu} f+f \partial_{\mu} \varepsilon\right) .
$$

Then

$$
\frac{\delta \mathcal{L}}{\delta\left(\partial_{\mu} \varepsilon\right)}=f \frac{\delta \mathcal{L}}{\delta\left(\partial_{\mu} \phi\right)}
$$

so that the Noether current can also be written as

$$
j^{\mu}=\frac{\delta \mathcal{L}}{\delta\left(\partial_{\mu} \varepsilon\right)} .
$$


Since in quantum mechanics the time development of an operator $\hat{Q}$ is given by

$$
\frac{d \hat{Q}}{d t}=i[\hat{H}, \hat{Q}]
$$

we see that such a conserved charge must commute with the Hamiltonian. Now in general the vacuum (or lowest energy state) of such a theory, which satisfies $\hat{H}\left|0>=E_{0}\right| 0>$, is unique and has the property $\hat{Q}|0>=| 0>$ since $\hat{H}(\hat{Q} \mid 0>$ )$=\hat{Q}(\hat{H} \mid 0>)=E_{0} \hat{Q} \mid 0>$. In this case we say that the symmetry is realized in a Wigner-Weyl fashion and there will exist in general a set of degenerate excited states which mix with each other under application of the symmetry charge. A familiar example of a Wigner-Weyl symmetry is isospin or $\mathrm{SU}(2)$ invariance. Because this is an (approximate) symmetry of the Hamiltonian, particles appear in multiplets such as $p, n$ or $\pi^{+} \pi^{0} \pi^{-}$having the same spinparity and (almost) the same mass and transform into one another under application of the isospin charges $\vec{I}$. However, this is not the only situation which occurs in nature. It is also possible (and in fact often the case) that the ground state of a system does not have the same symmetry as does the Hamiltonian, in which case we say that the symmetry is realized in a NambuGoldstone fashion and is "spontaneously broken". This phenomenon is actually a familiar one from classical mechanics and was first studied by Euler in the context of a rod under compression and by Jacobi in the context of a rotating earth.

In fact the idea exact symmetry and therefore exact conservation laws is not the usual one in nature. Rather it is common to have to deal with approximate symmetries which would be exact only in some hypothetical universe which is not our own - in our world such symmetries will be seen to be broken in some fashion. In spite of this, such broken symmetries are of great importance and by their study we will be able to learn much about the underlying interactions.

\subsection{Symmetry Breaking}

In general there exist in physics only three possible mechanisms for symmetry breaking

- explicit symmetry breaking

- spontaneous symmetry breaking

- quantum mechanical symmetry breaking 
and in this section we study examples of each:

\section{Explicit Symmetry Breaking}

First consider a simple harmonic oscillator of frequency $\omega_{0}$ described by the Lagrangian

$$
L=\frac{1}{2} m \dot{x}^{2}-\frac{1}{2} m \omega_{0}^{2} x^{2} .
$$

which is explicitly invariant under spatial inversion- $-x \rightarrow-x-$ since

$$
V_{0}(x)=-\frac{1}{2} m \omega_{0}^{2} x^{2}=-\frac{1}{2} m \omega^{2}(-x)^{2}=V_{0}(-x)
$$

Thus it is clear from symmetry considerations that the equilibrium location $x_{E}$, which is determined by the condition $[\partial L / \partial x]\left(x_{E}\right)=0$, must occur at $x_{E}=0$, since the equilibrium position should also manifest this symmetry.

Now, however, consider what happens if we add an term $V_{1}(x)=\lambda x$ i.e. a constant force, to the Lagrangian. The new Lagrangian is

$$
L=\frac{1}{2} m \dot{x}^{2}-\frac{1}{2} m \omega_{0}^{2} x^{2}+\lambda x
$$

which describes a displaced oscillator. This new Lagrangian is not invariant under spatial inversion, and consequently the new equilibrium location$x_{E}=\lambda / m \omega^{2} \neq 0$ - is no longer required to be at the origin. This is an example of explicit symmetry breaking wherein the symmetry violation is manifested in the Lagrangian itself.

\section{Spontaneous Symmetry Breaking}

As our second example, consider a hoop rotating in the earth's gravitational field about a vertical axis 10 Attached to the hoop is a bead which can slide along the circumference without friction. The lagrangian $L$ for the system is then

$$
L=\frac{1}{2} m\left(R^{2} \dot{\theta}^{2}+\omega^{2} R^{2} \sin ^{2} \theta\right)+m g R \cos \theta,
$$

where $\theta$ measures the angular displacement of the bead from the nadir. $L$ is clearly symmetric under the angular parity transformation $L(\theta)=L(-\theta)$, but the equilibrium condition for the bead is found to be

$$
\frac{\partial L}{\partial \theta}=m \omega^{2} R^{2} \sin \theta\left(\cos \theta-\frac{g}{\omega^{2} R}\right)=0 .
$$


which is somewhat more complex than the displaced oscillator considered above. For slow rotation-i.e for $\omega^{2}<\frac{g}{R}$, we have $\cos \theta-\frac{g}{\omega^{2} R} \neq 0$, so that the ground (equilibrium) state configuration is given by $\theta_{E}=0$ as expected from symmetry considerations. However, if we proceed to higher angular velocities such that $\omega^{2}>\frac{g}{R}$ then the bead finds equilibrium at $\theta_{E}= \pm \cos ^{-1} \frac{g}{\omega^{2} R}$, where the choice of + vs. - is not determined by the physics but rather by the history of motion of the system as the critical angular velocity was reached. Note that neither of these equilibrium positions exhibits the symmetry of the underlying potential, which is invariant under the exchange of $\theta$ and $-\theta$. This is an example of spontaneous symmetry breaking, wherein the Lagrangian of a system possess a symmetry, but this symmetry is broken by the ground (equilibrium) state of the system.

\section{Quantum Mechanical Symmetry Breaking}

The third type of symmetry breaking is the least familiar to most physicists because it has no classical analog. It is called "quantum mechanical" or "anomalous" symmetry breaking and occurs when the classical Lagrangian of a system possesses a symmetry, but the symmetry broken in the process of quantization. As the simplest example and the only one (of which I am aware) that does not involve quantum field theory - just quantum mechanics!-consider a free particle, for which the stationary state Schrödinger equation is 11

$$
-\frac{1}{2 m} \nabla^{2} \psi=E \psi \equiv \frac{k^{2}}{2 m} \psi,
$$

A partial wave solution in polar coordinates is

$$
\psi(\vec{r})=\frac{1}{r} \chi_{k}(r) P_{l}(\cos \theta),
$$

where $\chi_{k}(\vec{r})$ satisfies the radial Schrödinger equation

$$
\left(-\frac{d^{2}}{d r^{2}}+\frac{l(l+1)}{r^{2}}+k^{2}\right) \chi_{k}(r)=0 .
$$

Here the central piece in the above differential operator is the well-known "centrifugal potential." By inspection the radial Schrödinger equation is invariant under a "scale transformation"

$$
r \rightarrow \lambda r \quad k \rightarrow \frac{1}{\lambda} k
$$


This scale invariance has an important physical consequence, which can be seen if we expand a plane wave solution in terms of incoming and outgoing partial waves

$$
e^{i k z} \stackrel{r \rightarrow \infty}{\longrightarrow} \frac{1}{2 i k r} \sum_{l}(2 l+1) P_{l}(\cos \theta)\left(e^{i k r}-e^{-i(k r-l \pi)}\right),
$$

We observe that in each partial wave the incoming and outgoing component of the wavefunction differ by the centrifugal phase shift $l \pi$. This phase shift must be independent of energy via scale invariance.

If we place the free particle in a potential $V(\vec{r})$ then the scale invariance is broken. The corresponding wave function expanded in partial waves then becomes

$$
\psi^{(+)}(\vec{r}) \stackrel{r \rightarrow \infty}{\longrightarrow} \frac{1}{2 i k r} \sum_{l}(2 l+1) P_{l}(\cos \theta)\left(e^{i\left(k r+2 \delta_{l}(k)\right)}-e^{-i(k r-l \pi)}\right)
$$

Usually this is written as

$$
\psi^{(+)}(\vec{r})=e^{i k x}+\frac{e^{i k r}}{r} f_{k}(\theta)
$$

where the scattering amplitude is defined by

$$
f_{k}(\theta)=\sum_{l}(2 l+1) \frac{e^{2 i \delta_{l}(k)}-1}{2 i k} P_{l}(\cos \theta) .
$$

Of course, the phase shifts $\delta_{l}(k)$ of various angular momenta $l$ now depend on energy, but this is to be expected since the scale invariance no longer obtains.

One can generalize the scattering formalism to two dimensions, in which case we obtain for the scattering wave function

$$
\psi^{(+)}(\vec{r}) \stackrel{r \rightarrow \infty}{\longrightarrow} e^{i k z}+\frac{1}{\sqrt{r}} e^{i\left(k r+\frac{\pi}{4}\right)} f_{k}(\theta)
$$

and for the scattering amplitude

$$
f_{k}(\theta)=-i \sum_{m=-\infty}^{\infty} \frac{e^{2 i \delta_{m}(k)}-1}{\sqrt{2 \pi k}} e^{i m \theta}
$$

where we expand in terms of exponentials $e^{i m \theta}$ rather than Legendre polynomials. What is special about two dimensions is that it is possible to introduce a scale invariant potential

$$
V(\vec{r})=g \delta^{2}(\vec{r})
$$


The associated differential scattering cross section is found to be12

$$
\frac{d \sigma}{d \Omega} \propto \frac{\pi}{2 k} \frac{1}{\left(\ln \frac{k}{\mu}^{2}\right)} .
$$

which is somewhat of a surprise. Indeed since the cross section is isotropic, the scattering is pure $m=0$, corresponding to a phase shift

$$
\cot \delta_{0}(k)=\frac{1}{\pi} \ln \frac{k^{2}}{\mu^{2}}-\frac{2}{g},
$$

which depends on $k$ - scale invariance has been broken as a result of quantization. Although this should not be completely unexpected (indeed while at the classical level non-zero impact parameter means no scattering, in quantum mechanics this is not the case because of the non-zero deBroglie wavelength), still the "physics" of this result is not completely clear.

\section{Chiral Symmetry}

In order to understand the relevance of spontaneous symmetry breaking within QCD, we must introduce the idea of "chirality," defined by the operators

$$
\Gamma_{L, R}=\frac{1}{2}\left(1 \pm \gamma_{5}\right)=\frac{1}{2}\left(\begin{array}{cc}
1 & \mp 1 \\
\mp 1 & 1
\end{array}\right)
$$

which project left- and right-handed components of the Dirac wavefunction via

$$
\psi_{L}=\Gamma_{L} \psi \quad \psi_{R}=\Gamma_{R} \psi \quad \text { with } \quad \psi=\psi_{L}+\psi_{R}
$$

In terms of these chirality states the quark component of the QCD Lagrangian can be written as

$$
\bar{q}(i \not D-m) q=\bar{q}_{L} i \not D q_{L}+\bar{q}_{R} i \not D q_{R}-\bar{q}_{L} m q_{R}-\bar{q}_{R} m q_{L}
$$

The reason that these chirality states are called left- and right-handed can be seen by examining helicity eigenstates of the free Dirac equation. In the high energy (or massless) limit we note that

$$
u(p)=\sqrt{\frac{E+m}{2 E}}\left(\begin{array}{c}
\chi \\
\frac{\vec{\sigma} \cdot \vec{p}}{E+m} \chi
\end{array}\right) \stackrel{E \gg m}{\sim} \sqrt{\frac{1}{2}}\left(\begin{array}{c}
\chi \\
\vec{\sigma} \cdot \hat{p} \chi
\end{array}\right)
$$

Left- and right-handed helicity eigenstates then can be identified as

$$
u_{L}(p) \sim \sqrt{\frac{1}{2}}\left(\begin{array}{c}
\chi \\
-\chi
\end{array}\right), \quad u_{R}(p) \sim \sqrt{\frac{1}{2}}\left(\begin{array}{c}
\chi \\
\chi
\end{array}\right)
$$


But

$$
\begin{array}{cc}
\Gamma_{L} u_{L}=u_{L} & \Gamma_{R} u_{L}=0 \\
\Gamma_{R} u_{R}=u_{R} & \Gamma_{L} u_{R}=0
\end{array}
$$

so that in this limit chirality is identical with helicity-

$$
\Gamma_{L, R} \sim \text { helicity! }
$$

With this background, we now return to QCD and observe that in the limit as $m \rightarrow 0$

$$
\mathcal{L}_{\mathrm{QCD}} \rightarrow \bar{q}_{L} i \not D q_{L}+\bar{q}_{R} i \not D q_{R}
$$

would be invariant under independent global left- and right-handed rotations

$$
q_{L} \rightarrow \exp \left(i \sum_{j} \lambda_{j} \alpha_{j}\right) q_{L}, \quad q_{R} \rightarrow \exp \left(i \sum_{j} \lambda_{j} \beta_{j}\right) q_{R}
$$

(Of course, in this limit the heavy quark component is also invariant, but since $m_{c, b, t}>>\Lambda_{\mathrm{QCD}}$ it would be silly to consider this as even an approximate symmetry in the real world.) This invariance is called $S U(3)_{L} \otimes S U(3)_{R}$ or chiral $S U(3) \times S U(3)$. Continuing to neglect the light quark masses, we see that in a chiral symmetric world one would expect to have have sixteen - eight left-handed and eight right-handed-conserved Noether currents

$$
\bar{q}_{L} \gamma_{\mu} \frac{1}{2} \lambda_{i} q_{L}, \quad \bar{q}_{R} \gamma_{\mu} \frac{1}{2} \lambda_{i} q_{R}
$$

Equivalently, by taking the sum and difference we would have eight conserved vector and eight conserved axial vector currents

$$
V_{\mu}^{i}=\bar{q} \gamma_{\mu} \frac{1}{2} \lambda_{i} q, \quad A_{\mu}^{i}=\bar{q} \gamma_{\mu} \gamma_{5} \frac{1}{2} \lambda_{i} q
$$

In the vector case, this is just a simple generalization of isospin $(\mathrm{SU}(2))$ invariance to the case of $\mathrm{SU}(3)$. There exist eight $\left(3^{2}-1\right)$ time-independent charges

$$
F_{i}=\int d^{3} x V_{0}^{i}(\vec{x}, t)
$$

and there exist various supermultiplets of particles having identical spin-parity and (approximately) the same mass in the configurations - singlet, octet, decuplet, etc. demanded by $\mathrm{SU}(3)$ invariance.

If chiral symmetry were realized in the conventional fashion one would expect there also to exist corresponding nearly degenerate but opposite parity 
states generated by the action of the time-independent axial charges $F_{i}^{5}=$ $\int d^{3} x A_{0}^{i}(\vec{x}, t)$ on these states. Indeed since

$$
\begin{aligned}
H|P\rangle & =E_{P}|P\rangle \\
H\left(Q_{5}|P\rangle\right) & =Q_{5}(H|P\rangle)=E_{P}\left(Q_{5}|P\rangle\right)
\end{aligned}
$$

we see that $Q_{5}|P\rangle$ must also be an eigenstate of the Hamiltonian with the same eigenvalue as $|P\rangle$, which would seem to require the existence of parity doublets. However, experimentally this does not appear to be the case. Indeed although the $J^{p}=\frac{1}{2}^{+}$nucleon has a mass of about $1 \mathrm{GeV}$, the nearest $\frac{1}{2}^{-}$ resonce lies nearly $600 \mathrm{MeV}$ higher in energy. Likewise in the case of the $0^{-}$ pion which has a mass of about $140 \mathrm{MeV}$, the nearest corresponding $0^{+}$state (if it exists at all) is nearly $700 \mathrm{MeV}$ or so higher in energy.

\subsection{Goldstone's Theorem}

One can resolve this apparent paradox by postulating that parity-doubling is avoided because the axial symmetry is spontaneously broken. Then according to a theorem due to Goldstone, when a continuous symmetry is broken in this fashion there must also be generated a massless boson having the quantum numbers of the broken generator - in this case a pseudoscalar - and when the axial charge acts on a single particle eigenstate one does not get a single particle eigenstate of opposite parity in return 13 Rather one generates one or more of these massless pseudoscalar bosons

$$
Q_{5}|P\rangle \sim|P a\rangle+\cdots
$$

and the interactions of such "Goldstone bosons" to each other and to other particles is found to vanish as the four-momentum goes to zero.

In order to see how the corresponding situation develops in $\mathrm{RCD}$, it is useful to study a simple pedagogical example - a scalar field theory 4

$$
\mathcal{L}=\partial_{\mu} \phi^{*} \partial^{\mu} \phi-V\left(\phi^{*} \phi\right) \quad \text { with } \quad V(x)=\frac{\lambda}{4}\left(x-\frac{\mu^{2}}{\lambda}\right)^{2}
$$

which is obviously invariant under the global U(1) (phase) transformation $\phi \rightarrow e^{i \alpha} \phi$. The vacuum (lowest energy) state of the system can be found by minimizing the Hamiltonian density

$$
\mathcal{H}=\dot{\phi}^{*} \dot{\phi}+\vec{\nabla} \phi^{*} \cdot \vec{\nabla} \phi+V\left(\phi^{*} \phi\right)
$$


Since this is the sum of positive definite terms, the vacuum state is easily seen to be $\phi=v=\mu / \sqrt{\lambda}$, where $\mathrm{U}(1)$ symmetry has been used in order to choose $v$ as real. Of course, once this is done the $\mathrm{U}(1)$ symmetry is broken - spontaneous symmetry breaking has takne place - and Goldstone's theorem is applicable.

In order to see how this comes about we select as independent fields the real and imaginary components of $\phi-\rho \equiv \sqrt{2}(\operatorname{Re} \phi-v), \chi \equiv \sqrt{2} \operatorname{Im} \phi$-in terms of which the Lagrangian density becomes

$$
\mathcal{L}=\frac{1}{2} \partial_{\mu} \rho \partial^{\mu} \rho+\frac{1}{2} \partial_{\mu} \chi \partial^{\mu} \chi-\frac{1}{2} \mu^{2} \rho^{2}-\frac{\lambda}{16}\left(\rho^{2}+\chi^{2}\right)^{2}-\frac{\mu \sqrt{\lambda}}{2 \sqrt{2}} \rho\left(\rho^{2}+\chi^{2}\right)
$$

We observe that the field $\chi$ is massless - this is the Goldstone mode - while the field $\rho$ has a mass $\mu$. The Noether current

$$
j_{\mu}=-i\left(\phi^{*} \partial_{\mu} \phi-\partial_{\mu} \phi^{*} \phi\right)=\sqrt{2} v \partial_{\mu} \chi+\rho \partial_{\mu} \chi-\chi \partial_{\mu} \rho
$$

possesses a nonzero matrix element between $\chi$ and the vacuum

$$
<\chi(p)\left|j_{\mu}\right| 0>=\sqrt{2} v_{i p} e^{-i p \cdot x}
$$

provided that $v \neq 0$. Also there exist complicated self interactions as well as mutual interactions between $\rho$ and $\sigma$. However, if we calculate the tree-level amplitude for $\rho \chi$ scattering, using the diagrams illustrated in figure 4 we find

$$
\begin{aligned}
\operatorname{Amp}(\rho(q)+\chi(p) & \left.\rightarrow \rho\left(q^{\prime}\right)+\chi\left(p^{\prime}\right)\right)=\frac{\lambda}{2}+\frac{3}{2} \lambda^{2} v^{2} \frac{1}{\left(p+p^{\prime}\right)^{2}-\mu^{2}} \\
& +\frac{1}{2} \lambda^{2} v^{2}\left(\frac{1}{(p+q)^{2}}+\frac{1}{\left(p-q^{\prime}\right)^{2}}\right),
\end{aligned}
$$

and in the soft momentum limit for the Goldstone bosons- $p, p^{\prime} \rightarrow 0$ - we find that

$$
\lim _{p, p^{\prime} \rightarrow 0} \operatorname{Amp}=\frac{\lambda}{2}-\frac{3}{2} \frac{\lambda^{2} v^{2}}{\mu^{2}}+\frac{\lambda^{2} v^{2}}{\mu^{2}}=0
$$

i.e., the amplitude vanishes, as asserted above.

Thus our toy model certainly has all the right stuff, but our representation of the fields is not the optimal one in order to display the Goldstone properties. Instead it is advantageous to utilize a polar co-ordinate representation in which the Goldstone mode appears in the guise of a local U(1) transformation$\phi=\left(v+\sqrt{\frac{1}{2}} \xi\right) \exp i \theta / v \sqrt{2}$ - whereby the Lagrangian density assumes the form

$$
\mathcal{L}=\frac{1}{2} \partial_{\mu} \xi \partial^{\mu} \xi+\frac{1}{2}\left(1+\frac{1}{\sqrt{2}} \frac{\xi}{v}\right)^{2} \partial_{\mu} \theta \partial^{\mu} \theta-\frac{1}{2} \mu^{2} \xi^{2}-\frac{\mu \lambda}{2 \sqrt{2}} \xi^{3}-\frac{\lambda}{16} \xi^{4}
$$



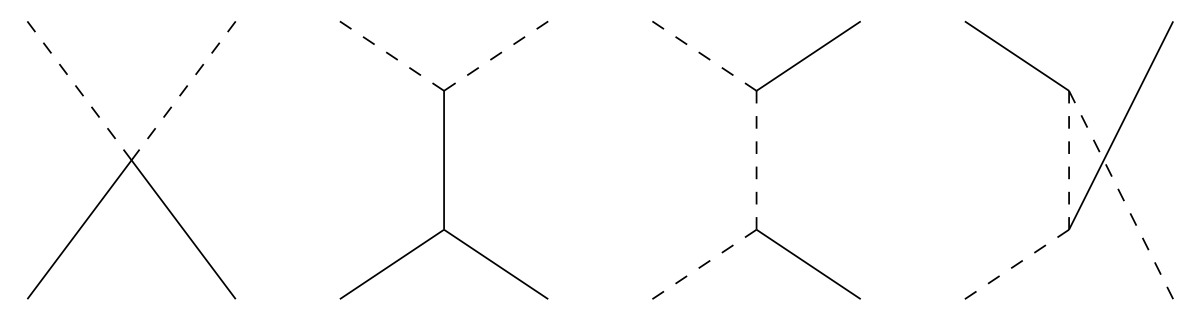

Figure 4: Toy model $\rho \chi$ scattering diagrams.

We see in this form that $\theta$ is the massless Goldstone field, while the field $\xi$ has mass $\mu$. The Noether current

$$
j_{\mu}=\sqrt{2} v\left(1+\sqrt{\frac{1}{2}} \frac{\xi}{v}\right)^{2} \partial_{\mu} \theta
$$

clearly has a nonzero vacuum-Goldstone matrix element which agrees with Eq. 66. However, what is particularly useful about this representation is the feature that the Goldstone modes couple only through derivative coupling. Thus the feature that any such interactions must vanish in the soft momentum limit is displayed explicitly, making Eq. 68 trivial.

Now back to QCD: According to Goldstone's argument, one would expect there to exist eight massless pseudoscalar states - one for each spontaneously broken SU(3) axial generator, which would be the Goldstone bosons of QCD. Examination of the particle data tables reveals, however, that no such massless $0^{-}$particles exist. There do exist eight $0^{-}$particles- $\pi^{ \pm}, \pi^{0}, K^{ \pm}, K^{0}, \bar{K}^{0} \eta$ which are much lighter than their hadronic siblings. However, these states are certainly not massless and this causes us to ask what has gone wrong with what appears to be rigorous reasoning. The answer is found in the feature that our discussion thus far has neglected the piece of the QCD Lagrangian which is associated with quark mass and can be written in the form

$$
\mathcal{L}_{\mathrm{Q} C D}^{m}=-\left(\bar{u}_{L} u_{R}+\bar{u}_{R} u_{L}\right) m_{u}-\left(\bar{d}_{L} d_{R}+\bar{d}_{R} d_{L}\right) m_{d}
$$

Since clearly this term breaks the chiral symmetry-

$$
\begin{aligned}
\bar{q}_{L} q_{R} & \rightarrow \bar{q}_{L} \exp \left(-i \sum_{j} \lambda_{j} \alpha_{j}\right) \times \exp \left(i \sum_{j} \lambda_{j} \beta_{j}\right) q_{R} \\
& \neq \bar{q}_{L} q_{R}
\end{aligned}
$$

—we have a violation of the conditions under which Goldstone's theorem aplies. 
The associated pseudoscalar bosons are not required to be massless

$$
m_{G}^{2} \neq 0
$$

but since their mass arises only from the breaking of the symmetry the various "would-be" Goldstone masses are expected to be proportional to the symmetry breaking parameters

$$
m_{G}^{2} \propto m_{u}, m_{d}, m_{s}
$$

To the extent that such quark masses are small the eight pseudoscalar masses are not required to be massless, merely much lighter than other hadronic masses in the spectrum, as found in nature.

\subsection{Effective Chiral Lagrangian}

The existence of a set of particles - the pseudoscalar mesons - which are notably less massive than other hadrons suggests the possibility of generating an effective field theory which correctly incorporates the chiral symmetry of the underlying QCD Lagrangian in describing the low energy interactions of these would-be Goldstone particles. As found in our pedagogical example, and in a homework problem, this can be formulated in a variety of ways, but the most transparent is done by including the Goldstone modes in terms of the argument of an exponential $U=\exp (i \vec{\tau} \cdot \phi / v)$ such that under the chiral transformations

$$
\begin{aligned}
\psi_{L} & \rightarrow L \psi_{L} \\
\psi_{R} & \rightarrow R \psi_{R}
\end{aligned}
$$

we have

$$
U \rightarrow L U R^{\dagger}
$$

Then a form such as

$$
\operatorname{Tr} \partial^{\mu} U \partial_{\mu} U^{\dagger} \rightarrow \operatorname{Tr} L \partial^{\mu} U R^{\dagger} R \partial_{\mu} U^{\dagger} L^{\dagger}=\operatorname{Tr} \partial^{\mu} U \partial_{\mu} U^{\dagger}
$$

is invariant under chiral rotations and can be used as part of the effective Lagrangian. However, this form is also not one which we can use in order to realistically describe Goldstone interactions in Nature since according to Goldstone's theorem a completely invariant Lagrangian must also have zero pion mass, in contradiction to experiment. We must include a term which uses the quark masses to generate chiral symmetry breaking and thereby non-zero pion mass. 
We infer then that the lowest order effective chiral Lagrangian can be written as

$$
\mathcal{L}_{2}=\frac{v^{2}}{4} \operatorname{Tr}\left(\partial_{\mu} U \partial^{\mu} U^{\dagger}\right)+\frac{m_{\pi}^{2}}{4} v^{2} \operatorname{Tr}\left(U+U^{\dagger}\right) .
$$

where the subscript 2 indicates that we are working at two-derivative order or one power of chiral symmetry breaking-i.e. $m_{\pi}^{2}$. This Lagrangian is also unique -if we expand to lowest order in $\vec{\phi}$

$$
\operatorname{Tr} \partial_{\mu} U \partial^{\mu} U^{\dagger}=\operatorname{Tr} \frac{i}{v} \vec{\tau} \cdot \partial_{\mu} \vec{\phi} \times \frac{-i}{v} \vec{\tau} \cdot \partial^{\mu} \vec{\phi}=\frac{2}{v^{2}} \partial_{\mu} \vec{\phi} \cdot \partial^{\mu} \vec{\phi}
$$

we reproduce the free pion Lagrangian, as required,

$$
\mathcal{L}_{2}=\frac{1}{2} \partial_{\mu} \vec{\phi} \cdot \partial^{\mu} \vec{\phi}-\frac{1}{2} m_{\pi}^{2} \vec{\phi} \cdot \vec{\phi}+\mathcal{O}\left(\phi^{4}\right) .
$$

At the $\mathrm{SU}(3)$ level, including a generalized chiral symmetry breaking term, there is even predictive power - one has

$$
\begin{aligned}
\frac{v^{2}}{4} \operatorname{Tr} \partial_{\mu} U \partial^{\mu} U^{\dagger}=\frac{1}{2} \sum_{j=1}^{8} \partial_{\mu} \phi_{j} \partial^{\mu} \phi_{j}+\cdots \\
\frac{v^{2}}{4} \operatorname{Tr} 2 B_{0} m\left(U+U^{\dagger}\right)=\text { const. }-\frac{1}{2}\left(m_{u}+m_{d}\right) B_{0} \sum_{j=1}^{3} \phi_{j}^{2} \\
-\frac{1}{4}\left(m_{u}+m_{d}+2 m_{s}\right) B_{0} \sum_{j=4}^{7} \phi_{j}^{2}-\frac{1}{6}\left(m_{u}+m_{d}+4 m_{s}\right) B_{0} \phi_{8}^{2}+\cdots
\end{aligned}
$$

where $B_{0}$ is a constant and $m$ is the quark mass matrix. We can then identify the meson masses as

$$
\begin{aligned}
m_{\pi}^{2} & =2 \hat{m} B_{0} \\
m_{K}^{2} & =\left(\hat{m}+m_{s}\right) B_{0} \\
m_{\eta}^{2} & =\frac{2}{3}\left(\hat{m}+2 m_{s}\right) B_{0},
\end{aligned}
$$

where $\hat{m}=\frac{1}{2}\left(m_{u}+m_{d}\right)$ is the mean light quark mass. This system of three equations is overdetermined, and we find by simple algebra

$$
3 m_{\eta}^{2}+m_{\pi}^{2}-4 m_{K}^{2}=0
$$


which is the Gell-Mann-Okubo mass relation and is well-satisfied experimentally 15

\section{Currents}

Since under a

$$
\text { Vector, Axial transformation: } \alpha_{L}= \pm \alpha_{R}
$$

we have

$$
U \rightarrow \mathrm{LUR}^{\dagger} \stackrel{V}{\simeq} U+i\left[\sum_{j} \alpha_{j} \lambda_{j}, U\right] \stackrel{A}{\simeq} U+i\left\{\sum_{j} \alpha_{j} \lambda_{j}, U\right\} .
$$

which leads to the vector and axial-vector currents

$$
\{V, A\}_{\mu}^{k}=-i \frac{v^{2}}{4} \operatorname{Tr} \lambda^{k}\left(U^{\dagger} \partial_{\mu} U \pm U \partial_{\mu} U^{\dagger}\right)
$$

At this point the constant $v$ can be identified by use of the axial current. In $\mathrm{SU}(2)$ we find

$$
U^{\dagger} \partial_{\mu} U-U \partial_{\mu} U^{\dagger}=2 i \frac{1}{v} \vec{\tau} \cdot \partial_{\mu} \vec{\phi}+\cdots
$$

so that

$$
A_{\mu}^{k}=i \frac{v^{2}}{4} \operatorname{Tr} \tau^{k} 2 i \frac{1}{v} \vec{\tau} \cdot \partial_{\mu} \vec{\phi}+\cdots=-v \partial_{\mu} \phi^{k}+\cdots .
$$

If we set $k=1-i 2$ then this represents the axial-vector component of the $\Delta S=0$ charged weak current and

$$
A_{\mu}^{1-i 2}=-v \partial_{\mu} \phi^{1-i 2}=-\sqrt{2} v \partial_{\mu} \phi^{-} .
$$

Comparing with the conventional definition

$$
\left\langle 0\left|A_{\mu}^{1-i 2}(0)\right| \pi^{+}(p)\right\rangle=i \sqrt{2} F_{\pi} p_{\mu},
$$

we find that, to lowest order in chiral symmetry, $v=F_{\pi}$, where $F_{\pi}=92.4$ $\mathrm{MeV}$ is the pion decay constant $1 \mathrm{~d}$

Likewise in $\mathrm{SU}(2)$, we note that

$$
U^{\dagger} \partial_{\mu} U+U \partial_{\mu} U^{\dagger}=\frac{2 i}{v^{2}} \vec{\tau} \cdot \vec{\phi} \times \partial_{\mu} \vec{\phi}+\cdots,
$$


so that the vector current is

$$
\begin{aligned}
V_{\mu}^{k} & =-i \frac{v^{2}}{4} \operatorname{Tr} \tau^{k} \frac{2 i}{v^{2}} \vec{\tau} \vec{\phi} \times \partial_{\mu} \vec{\phi}+\cdots \\
& =\left(\vec{\phi} \times \partial_{\mu} \vec{\phi}\right)^{k}+\cdot
\end{aligned}
$$

We can identify $V_{\mu}^{k}$ as the (isovector) electromagnetic current by setting $k=3$ so that

$$
V_{\mu}^{\mathrm{em}}=\phi^{+} \partial_{\mu} \phi^{-}-\phi^{-} \partial_{\mu} \phi^{+}+\cdots
$$

Comparing with the conventional definition

$$
\left\langle\pi^{+}\left(p_{2}\right)\left|V_{\mu}^{\mathrm{em}}(0)\right| \pi^{+}\left(p_{1}\right)\right\rangle=F_{1}\left(q^{2}\right)\left(p_{1}+p_{2}\right)_{\mu},
$$

we identify the pion form factor $-F_{1}\left(q^{2}\right)=1$. Thus to lowest order in chiral symmetry the pion has unit charge but is pointlike and structureless. We shall see below how to insert structure.

\section{$\pi \pi$ Scattering}

At two derivative level we can generate additional predictions by extending our analysis to the case of $\pi \pi$ scattering. Expanding $\mathcal{L}_{2}$ to order $\vec{\phi}^{4}$ we find

$$
\mathcal{L}_{2}: \phi^{4}=\frac{1}{6 v^{2}} \vec{\phi}^{2} \vec{\phi} \cdot \square \vec{\phi}+\frac{1}{2 v^{2}}\left(\vec{\phi} \cdot \partial_{\mu} \vec{\phi}\right)^{2}+\frac{m_{\pi}^{2}}{24 v^{2}} \vec{\phi}^{4}
$$

which yields for the pi-pi $T$ matrix

$$
\begin{aligned}
T\left(q_{a}, q_{b} ; q_{c}, q_{d}\right)= & \frac{1}{F_{\pi}^{2}}\left[\delta^{a b} \delta^{c d}\left(s-m_{\pi}^{2}\right)+\delta^{a b} \delta^{b d}\left(t-m_{\pi}^{2}\right)+\delta^{a d} \delta^{b c}\left(u-m_{\pi}^{2}\right)\right] \\
& -\frac{1}{3 F_{\pi}^{2}}\left(\delta^{a b} \delta^{c d}+\delta^{a c} \delta^{b d}+\delta^{a d} \delta^{b c}\right)\left(q_{a}^{2}+q_{b}^{2}+q_{c}^{2}+q_{d}^{2}-4 m_{\pi}^{2}\right)
\end{aligned}
$$

Defining more generally

$$
T_{\alpha \beta ; \gamma \delta}(s, t, u)=A(s, t, u) \delta_{\alpha \beta} \delta_{\gamma \delta}+A(t, s, u) \delta_{\alpha \gamma} \delta_{\beta \delta}+A(u, t, s) \delta_{\alpha \delta} \delta_{\beta \gamma},
$$

we can write the chiral prediction in terms of the more conventional isospin language by taking appropriate linear combination 3

$$
\begin{aligned}
& T^{0}(s, t, u)=3 A(s, t, u)+A(t, s, u)+A(u, t, s) \\
& T^{1}(s, t, u)=A(t, s, u)-A(u, t, s) \\
& T^{2}(s, t, u)=A(t, s, u)+A(u, t, s)
\end{aligned}
$$




\begin{tabular}{lrrr}
\hline \hline & Experimental & Lowest Order & \\
\hline$a_{0}^{0}$ & $0.26 \pm 0.05$ & 0.16 & First Two Orders $^{c}$ \\
$b_{0}^{0}$ & $0.25 \pm 0.03$ & 0.18 & 0.20 \\
$a_{0}^{2}$ & $-0.028 \pm 0.012$ & -0.045 & 0.26 \\
$b_{2}^{2}$ & $-0.082 \pm 0.008$ & -0.089 & -0.041 \\
$a_{1}^{1}$ & $0.038 \pm 0.002$ & 0.030 & -0.070 \\
$b_{1}^{1}-$ & 0 & 0.043 & 0.036 \\
$a_{2}^{0}$ & $(17 \pm 3) \times 10^{-4}$ & 0 & $20 \times 10^{-4}$ \\
$a_{2}^{2}$ & $(1.3 \pm 3) \times 10^{-4}$ & 0 & $3.5 \times 10^{-4}$ \\
\hline \hline
\end{tabular}

Table 1: The pion scattering lengths and slopes compared with predictions of chiral symmetry.

Partial wave amplitudes, projected out via

$$
T_{l}^{I}(s)=\frac{1}{64 \pi} \int_{-1}^{1} d(\cos \theta) P_{l}(\cos \theta) T^{I}(s, t, u),
$$

can be used to identify the associated scattering phase shifts via

$$
T_{l}^{I}(s)=\left(\frac{s}{s-4 m_{\pi}^{2}}\right)^{\frac{1}{2}} e^{i \delta_{l}^{I}} \sin \delta_{l}^{I} .
$$

Then from the lowest order chiral form

$$
A(s, t, u)=\frac{s-m_{\pi}^{2}}{F_{\pi}^{2}}
$$

we determine values for the pion scattering lengths and effective ranges

$$
\begin{aligned}
a_{0}^{0} & =\frac{7 m_{\pi}^{2}}{32 \pi F_{\pi}^{2}}, \quad a_{0}^{2}=-\frac{m_{\pi}^{2}}{16 \pi F_{\pi}^{2}}, \quad a_{1}^{1}=-\frac{m_{\pi}^{2}}{24 \pi F_{\pi}^{2}}, \\
b_{0}^{0} & =\frac{m_{\pi}^{2}}{4 \pi F_{\pi}^{2}}, \quad b_{0}^{2}=\frac{m_{\pi}^{2}}{8 \pi F_{\pi}^{2}},
\end{aligned}
$$

comparison of which with experimental numbers is shown in Table 1.

Despite the obvious success of this and other such predictions 17 it is clear that we do not really have at this point a satisfactory theory, since the strictures of unitarity are violated. Indeed, since we are working at tree level, all our 
amplitudes are real. However, unitarity of the S-matrix requires transition amplitudes to contain an imaginary component since

$$
\begin{gathered}
0=S^{\dagger} S-1=i\left(<f\left|T^{\dagger}\right| i>-<f|T| i>\right)+<f\left|T^{\dagger} T\right| i> \\
\text { i.e. } \quad 2 \operatorname{Im}<f|T| i>=\sum_{n}<f\left|T^{\dagger}\right| n><n|T| i>\neq 0
\end{gathered}
$$

The solution of such problems with unitarity are well known - the inclusion of loop corrections to these simple tree level calculations. Insertion of such loop terms removes the unitarity violations but comes with a high price - numerous divergences are introduced and this difficulty prevented progress ip this field for nearly a decade until a paper by Weinberg suggested the solution 18 One can deal with such divergences, just as in QED, by introducing phenomenologically determined counterterms into the Lagrangian in order to absorb the infinities. We see in the next section how this can be accomplished.

\section{Renormalization}

\subsection{Effective Chiral Lagrangian}

We can now apply Weinberg's solution to the effective chiral Lagrangian, Eq. 77. As noted above, when loop corrections are made to lowest order amplitudes in order to enforce unitarity, divergences inevitably arise. However, there is an important difference from the familiar case of QED in that the form of the divergences is different from their lower order counterparts-i.e. the theory is nonrenormalizable! The reason for this can be seen from a simple example. Thus consider pi-pi scattering. In lowest order there exists a tree level contribution from $\mathcal{L}_{2}$ which is $\mathcal{O}\left(p^{2} / F_{\pi}^{2}\right)$ where $p$ represents some generic external energy-momentum. The fact that $p$ appears to the second power is due to the feature that its origin is the two-derivative Lagrangian $\mathcal{L}_{2}$. Now suppose that pi-pi scattering is examined at one loop order. Since the scattering amplitude must still be dimensionless but now the amplitude involves a factor $1 / F_{\pi}^{4}$ the numerator must involve four powers of energy-momentum. Thus any counterterm which is included in order to absorb this divergence must be four-derivative in character. Gasser and Leutwyler have studied this problem and have written the most general form of such an order four counterterm in chiral SU(3) a:

$$
\mathcal{L}_{4}=\sum_{i=1}^{10} L_{i} \mathcal{O}_{i}=L_{1}\left[\operatorname{tr}\left(D_{\mu} U D^{\mu} U^{\dagger}\right)\right]+L_{2} \operatorname{tr}\left(D_{\mu} U D_{\nu} U^{\dagger}\right) \cdot \operatorname{tr}\left(D^{\mu} U D^{\nu} U^{\dagger}\right)
$$




$$
\begin{aligned}
& +L_{3} \operatorname{tr}\left(D_{\mu} U D^{\mu} U^{\dagger} D_{\nu} U D^{\nu} U^{\dagger}\right)+L_{4} \operatorname{tr}\left(D_{\mu} U D^{\mu} U^{\dagger}\right) \operatorname{tr}\left(\chi U^{\dagger}+U \chi^{\dagger}\right) \\
& +L_{5} \operatorname{tr}\left(D_{\mu} U D^{\mu} U^{\dagger}\left(\chi U^{\dagger}+U \chi^{\dagger}\right)\right)+L_{6}\left[\operatorname{tr}\left(\chi U^{\dagger}+U \chi^{\dagger}\right)\right]^{2} \\
& +L_{7}\left[\operatorname{tr}\left(\chi^{\dagger} U-U \chi^{\dagger}\right)\right]^{2}+L_{8} \operatorname{tr}\left(\chi U^{\dagger} \chi U^{\dagger}+U \chi^{\dagger} U \chi^{\dagger}\right) \\
& +i L_{9} \operatorname{tr}\left(F_{\mu \nu}^{L} D^{\mu} U D^{\nu} U^{\dagger}+F_{\mu \nu}^{R} D^{\mu} U^{\dagger} D^{\nu} U\right)+L_{10} \operatorname{tr}\left(F_{\mu \nu}^{L} U F^{R \mu \nu} U^{\dagger}\right)
\end{aligned}
$$

where the covariant derivative is defined via

$$
D_{\mu} U=\partial_{\mu} U+\left\{A_{\mu}, U\right\}+\left[V_{\mu}, U\right],
$$

the constants $L_{i}, i=1,2, \ldots 10$ are arbitrary (not determined from chiral symmetry alone) and $F_{\mu \nu}^{L}, F_{\mu \nu}^{R}$ are external field strength tensors defined via

$$
F_{\mu \nu}^{L, R}=\partial_{\mu} F_{\nu}^{L, R}-\partial_{\nu} F_{\mu}^{L, R}-i\left[F_{\mu}^{L, R}, F_{\nu}^{L, R}\right], \quad F_{\mu}^{L, R}=V_{\mu} \pm A_{\mu} .
$$

Now just as in the case of QED the bare parameters $L_{i}$ which appear in this Lagrangian are not physical quantities. Instead the experimentally relevant (renormalized) values of these parameters are obtained by appending to these bare values the divergent one-loop contributions having the form

$$
L_{i}^{r}=L_{i}-\frac{\gamma_{i}}{32 \pi^{2}}\left[\frac{-2}{\epsilon}-\ln (4 \pi)+\gamma-1\right]
$$

By comparing with experiment, Gasser and Leutwyler were able to determine empirical values for each of these ten parameters. While ten sounds like a rather large number, we shall see below that this picture is actually quite predictive. Typical values for the parameters are shown in Table 2.

The important question to ask at this point is why stop at order four derivativeas Clearly if two loop amplitudes from $\mathcal{L}_{2}$ or one-loop corrections from $\mathcal{L}_{4}$ are calculated, divergences will arise which are of six derivative character. Why not include these? The answer is that the chiral procedure represents an expansion in energy-momentum. Corrections to the lowest order (tree level) predictions from one loop corrections from $\mathcal{L}_{2}$ or tree level contributions from $\mathcal{L}_{4}$ are $\mathcal{O}\left(E^{2} / \Lambda_{\chi}^{2}\right)$ where $\Lambda_{\chi} \sim 4 \pi F_{\pi} \sim 1 \mathrm{GeV}$ is the chiral scale 19 Thus chiral perturbation theory is a low energy procedure. It is only to the extent that the energy is small compared to the chiral scale that it makes sense to truncate the expansion at the four-derivative level. Realistically this means that we deal with processes involving $E<500 \mathrm{MeV}$, and, as we shall describe below, for such reactions the procedure is found to work very well. 


\begin{tabular}{llc}
\hline \hline Coefficient & Value & Origin \\
\hline$L_{1}^{r}$ & $0.65 \pm 0.28$ & $\pi \pi$ scattering \\
$L_{2}^{r}$ & $1.89 \pm 0.26$ & and \\
$L_{3}^{r}$ & $-3.06 \pm 0.92$ & $K_{\ell 4}$ decay \\
$L_{5}^{r}$ & $2.3 \pm 0.2$ & $F_{K} / F_{\pi}$ \\
$L_{9}^{r}$ & $7.1 \pm 0.3$ & $\pi$ charge radius \\
$L_{10}^{r}$ & $-5.6 \pm 0.3$ & $\pi \rightarrow e \nu \gamma$ \\
\hline \hline
\end{tabular}

Table 2: Gasser-Leutwyler counterterms and the means by which they are determined.

Now Gasser and Leutwyler, besides giving the form of the $\mathcal{O}\left(p^{4}\right)$ chiral Lagrangian, have also performed the one loop integration and have written the result in a simple algebraic form. Users merely need to look up the result in their paper. However, in order to really understand what they have done, it is useful to study a simple example of a chiral perturbation theory calculation in order to see how it is performed and in order to understand how the experimental counterterm values are actually determined. We consider the pion electromagnetic form factor, which by Lorentz- and gauge-invariance has the structure

$$
\left\langle\pi^{+}\left(p_{2}\right)\left|J_{\mathrm{em}}^{\mu}\right| \pi^{+}\left(p_{1}\right)\right\rangle=F_{1}\left(q^{2}\right)\left(p_{1}+p_{2}\right)^{\mu}
$$

We begin by identifying the electromagnetic current as

$$
\begin{aligned}
J_{\mathrm{em}}^{\mu} & =-\frac{\partial \mathcal{L}}{\partial\left(e A_{\mu}\right)}=\left(\varphi \times \partial^{\mu} \varphi\right)_{3}\left[1-\frac{1}{3 F^{2}} \varphi \cdot \varphi+\mathcal{O}\left(\varphi^{4}\right)\right] \\
& +\left(\varphi \times \partial^{\mu} \varphi\right)_{3}\left[16 L_{4}+8 L_{5}\right] \frac{m_{\pi}^{2}}{F^{2}}+\frac{4 L_{9}}{F^{2}} \partial^{\nu}\left(\partial^{\mu} \varphi \times \partial_{\nu} \varphi\right)_{3}+\cdots
\end{aligned}
$$

where we have expanded to fourth order in the pseudoscalar fields. Defining

$$
\begin{aligned}
\delta_{j k} I\left(m^{2}\right) & =i \Delta_{F j k}(0)=\left\langle 0\left|\varphi_{j}(x) \varphi_{k}(x)\right| 0\right\rangle \\
I\left(m^{2}\right) & =\mu^{4-d} \int \frac{d^{d} k}{(2 \pi)^{d}} \frac{i}{k^{2}-m^{2}}=\frac{\mu^{4-d}}{(4 \pi)^{d / 2}} \Gamma\left(1-\frac{d}{2}\right)\left(m^{2}\right)^{\frac{d}{2}-1}, \\
\delta_{j k} I_{\mu \nu}\left(m^{2}\right) & =-\partial_{\mu} \partial_{\nu} i \Delta_{F j k}(0)=\left\langle 0\left|\partial_{\mu} \varphi_{j}(x) \partial_{\nu} \varphi_{k}(x)\right| 0\right\rangle \\
I_{\mu \nu}\left(m^{2}\right) & =\mu^{4-d} \int \frac{d^{d} k}{(2 \pi)^{d}} k_{\mu} k_{\nu} \frac{i}{k^{2}-m^{2}}=g_{\mu \nu} \frac{m^{2}}{d} I\left(m^{2}\right)
\end{aligned}
$$

we calculate the one loop correction shown in Figure 5a to be 


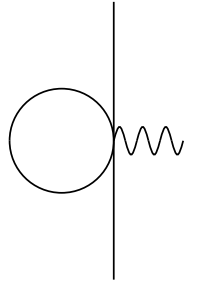

(a)

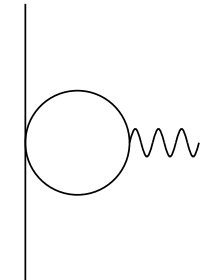

(b)

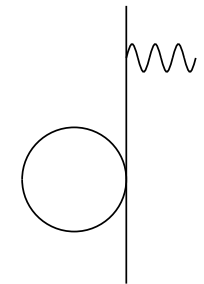

(c)

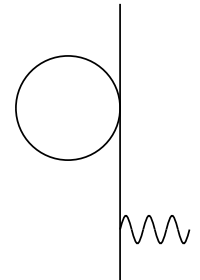

(d)

Figure 5: Loop corrections to the pion form factor.

$$
\left.J_{\mathrm{em}}^{\mu}\right|_{(4 a)}=-\frac{5}{3 F_{\pi}^{2}}\left(\varphi \times \partial^{\mu} \phi\right)_{3} I\left(m_{\pi}^{2}\right)
$$

We also need the one loop correction shown in Figure 5b. For this piece we require the form of the pi-pi scattering amplitude which arises from $\mathcal{L}_{2}$

$$
\left\langle\pi^{+}\left(k_{1}\right) \pi^{-}\left(k_{2}\right) \mid \pi^{+}\left(p_{1}\right) \pi^{-}\left(p_{2}\right)\right\rangle=\frac{i}{3 F_{0}^{2}}\left(2 m_{0}^{2}+p_{1}^{2}+p_{2}^{2}+k_{1}^{2}+k_{2}^{2}-3\left(p_{1}-k_{1}\right)^{2}\right)
$$

and we shall perform the loop integration using the method of dimensional regularization, which yields

$$
\begin{aligned}
\left\langle J_{\mathrm{em}}^{\mu}\right\rangle_{(5 b)} & =\frac{1}{\left(4 \pi F_{\pi}\right)^{2}}\left(p_{1}+p_{2}\right)^{\mu} \int_{0}^{1} d x\left(m_{\pi}^{2}-q^{2} x(1-x)\right) \\
& \times\left[\left(-\frac{2}{\epsilon}+\gamma-1-\ln 4 \pi\right)+\ln \frac{m_{\pi}^{2}-q^{2} x(1-x)}{\mu^{2}}\right]
\end{aligned}
$$

Performing the $\mathrm{x}$-integration we find, finally

$$
\begin{aligned}
\left\langle J_{\mathrm{em}}^{\mu}\right\rangle_{(5 b)}= & \frac{1}{\left(4 \pi F_{\pi}\right)^{2}}\left(p_{1}+p_{2}\right)^{\mu}\left\{\left(m_{\pi}^{2}-\frac{1}{6} q^{2}\right)\left[-\frac{2}{\epsilon}+\gamma-1-\ln 4 \pi+\ln \frac{m_{\pi}^{2}}{\mu^{2}}\right]\right. \\
& \left.+\frac{1}{6}\left(q^{2}-4 m_{\pi}^{2}\right) H\left(\frac{q^{2}}{m_{\pi}^{2}}\right)-\frac{1}{18} q^{2}\right\}
\end{aligned}
$$

where the function $\mathrm{H}(\mathrm{a})$ is given by

$$
H(a) \equiv \int_{0}^{1} d x \ln (1-a x(1-x))
$$




$$
= \begin{cases}2-2 \sqrt{\frac{4}{a}-1} \cot ^{-1} \sqrt{\frac{4}{a}-1} & (0<a<4) \\ 2+\sqrt{1-\frac{4}{a}}\left[\ln \frac{\sqrt{1-\frac{4}{a}}-1}{\sqrt{1-\frac{4}{a}}+1}+i \pi \theta(a-4)\right] & (\text { otherwise) }\end{cases}
$$

and contains the imaginary component required by unitarity.

We are not done yet, however, since we must also include mass and wavefunction effects - figs. $5 \mathrm{c}, 5 \mathrm{~d}$. In order to do so, we expand $\mathcal{L}_{2}$ to fourth order in $\varphi(x)$, and $\mathcal{L}_{4}$ to second order:

$$
\begin{aligned}
\mathcal{L}_{2}= & \frac{1}{2}\left[\partial^{\mu} \varphi \cdot \partial_{\mu} \varphi-m_{0}^{2} \varphi \cdot \varphi\right]+\frac{m_{0}^{2}}{24 F_{0}^{2}}(\varphi \cdot \varphi)^{2} \\
& +\frac{1}{6 F_{0}^{2}}\left[\left(\varphi \cdot \partial^{\mu} \varphi\right)\left(\varphi \cdot \partial_{\mu} \varphi\right)-(\varphi \cdot \varphi)\left(\partial^{\mu} \varphi \cdot \partial_{\mu} \varphi\right)\right]+\mathcal{O}\left(\varphi^{6}\right), \\
\mathcal{L}_{4}= & \frac{m_{0}^{2}}{F_{0}^{2}}\left[16 L_{4}+8 L_{5}\right] \frac{1}{2} \partial_{\mu} \varphi \cdot \partial^{\mu} \varphi \\
& -\frac{m_{0}^{2}}{F_{0}^{2}}\left[32 L_{6}+16 L_{8}\right] \frac{1}{2} m_{0}^{2} \varphi \cdot \varphi+\mathcal{O}\left(\varphi^{4}\right) .
\end{aligned}
$$

Performing the loop integrations on the $\phi^{4}(x)$ component of the above yields

$$
\begin{aligned}
\mathcal{L}_{\mathrm{eff}}= & \frac{1}{2} \partial^{\mu} \varphi \partial_{\mu} \varphi-\frac{1}{2} m_{0}^{2} \varphi \cdot \varphi+\frac{5 m_{\pi}^{2}}{12 F_{\pi}^{2}} I\left(m_{\pi}^{2}\right) \varphi \cdot \varphi \\
& +\frac{1}{6 F_{\pi}^{2}}\left(\delta_{i k} \delta_{j l}-\delta_{i j} \delta_{k l}\right) I\left(m_{\pi}^{2}\right)\left(\delta_{i j} \partial^{\mu} \varphi_{k} \partial_{\mu} \varphi_{l}+\delta_{k l} m_{\pi}^{2} \varphi_{i} \varphi_{j}\right) \\
& \left.+\frac{1}{2} \partial_{\mu} \varphi \cdot \partial^{\mu} \varphi \frac{m_{\pi}^{2}}{F_{\pi}^{2}}\left[16 L_{4}+8 L_{5}\right)\right]-\frac{1}{2} m_{\pi}^{2} \varphi \cdot \varphi \frac{m_{\pi}^{2}}{F_{\pi}^{2}}\left[32 L_{6}+16 L_{8}\right] \\
= & \frac{1}{2} \partial^{\mu} \varphi \cdot \partial_{\mu} \varphi\left[1+\left(16 L_{4}+8 L_{5}\right) \frac{m_{\pi}^{2}}{F_{\pi}^{2}}-\frac{2}{3 F_{\pi}^{2}} I\left(m_{\pi}^{2}\right)\right] \\
& -\frac{1}{2} m_{0}^{2} \varphi \cdot \varphi\left[1+\left(32 L_{6}+16 L_{8}\right) \frac{m_{\pi}^{2}}{F_{\pi}^{2}}-\frac{1}{6 F_{\pi}^{2}} I\left(m_{\pi}^{2}\right)\right]
\end{aligned}
$$

from which we can now read off the wavefunction renormalization term $Z_{\pi}$.

When this is done we find

$$
\begin{aligned}
Z_{\pi} F_{1}^{(\text {tree })}\left(q^{2}\right)= & {\left[1-\frac{8 m_{\pi}^{2}}{F_{\pi}^{2}}\left(2 L_{4}+L_{5}\right.\right.} \\
& \left.+\frac{m_{\pi}^{2}}{24 \pi^{2} F_{\pi}^{2}}\left\{-\frac{2}{\epsilon}+\gamma-1-\ln 4 \pi+\ln \frac{m_{\pi}^{2}}{\mu^{2}}\right\}\right]
\end{aligned}
$$




$$
\begin{aligned}
& \times\left[1+\frac{8 m_{\pi}^{2}}{F_{\pi}^{2}}\left(2 L_{4}+L_{5}\right)+2 q^{2} \frac{L_{9}}{F_{\pi}^{2}}\right] \\
= & {\left[1+\frac{m_{\pi}^{2}}{24 \pi^{2} F_{\pi}^{2}}\left(-\frac{2}{\epsilon}+\gamma-1-\ln 4 \pi+\ln \frac{m_{\pi}^{2}}{\pi^{2}}\right)+\frac{2 L_{9}}{F_{\pi}^{2}} q^{2}\right] }
\end{aligned}
$$

while from the loop diagrams given earlier

$$
\begin{aligned}
\left.F_{1}\left(q^{2}\right)\right|_{(5 a)}= & -\frac{5 m_{\pi}^{2}}{48 \pi^{2} F_{\pi}^{2}}\left\{-\frac{2}{\epsilon}+\gamma-1-\ln 4 \pi+\ln \frac{m_{\pi}^{2}}{\mu^{2}}\right\} \\
\left.F_{1}\left(q^{2}\right)\right|_{(5 b)}= & \frac{1}{16 \pi^{2} F_{\pi}^{2}}\left\{\left(m_{\pi}^{2}-\frac{1}{6} q^{2}\right)\left[-\frac{2}{\epsilon}+\gamma-1-\ln 4 \pi+\ln \frac{m_{\pi}^{2}}{\mu^{2}}\right]\right. \\
& \left.+\frac{1}{6}\left(q^{2}-4 m_{\pi}^{2}\right) H\left(\frac{q^{2}}{m_{\pi}^{2}}\right)-\frac{1}{18} q^{2}\right\}
\end{aligned}
$$

Adding everything together we have the final result, which when written in terms of the renormalized value $L_{9}^{(r)}$ is finite!

$$
F_{1}\left(q^{2}\right)=1+\frac{2 L_{9}^{(r)}}{F_{\pi}^{2}} q^{2}+\frac{1}{96 \pi^{2} F_{\pi}^{2}}\left[\left(q^{2}-4 m_{\pi}^{2}\right) H\left(\frac{q^{2}}{m_{\pi}^{2}}\right)-q^{2} \ln \frac{m_{\pi}^{2}}{\mu^{2}}-\frac{q^{2}}{3}\right]
$$

Expanding to lowest order in $q^{2}$ we find

$$
F_{1}\left(q^{2}\right)=1+q^{2}\left[\frac{2 L_{9}^{(r)}}{F_{\pi}^{2}}-\frac{1}{96 \pi^{2} F_{\pi}^{2}}\left(\ln \frac{m_{\pi}^{2}}{\mu^{2}}+1\right)\right]+\cdots
$$

which can be compared with the phenomenological description in terms of the pion charge radius

$$
F_{1}\left(q^{2}\right)=1+\frac{1}{6}\left\langle r_{\pi}^{2}\right\rangle q^{2}+\cdots
$$

By equating these two expressions and using the experimental value of the pion charge radius $-\left\langle r_{\pi}^{2}\right\rangle_{\exp }=(0.44 \pm 0.01) \mathrm{fm}$ - - we determine the value of the counterterm $L_{9}^{(r)}$ shown in Table 2.

As seen in Figure $\$$ this form gives a reasonable representation of the experimental pion form factor near threshold but deviates substantially from the empirical result as the $\rho$ resonance is approached. This is not surprising as any perturbative approach will be unable to reproduce resonant behavior. 


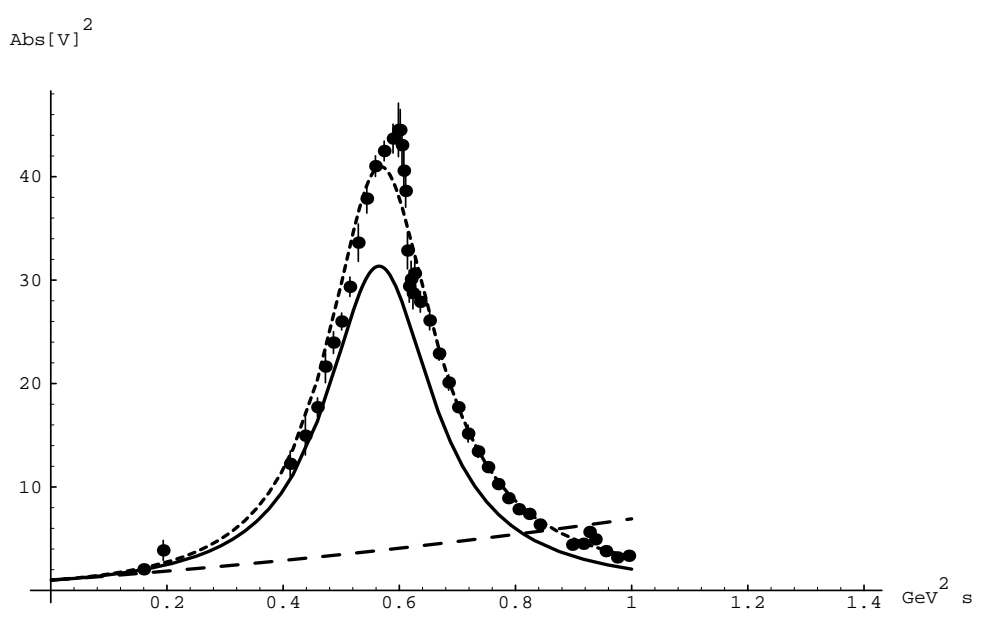

Figure 6: Calculations of the modulus of the pion form factor squared compared to experimental results. Here the solid line gives the result of the inverse amplitude method, while the dashed line gives the one loop chiral perturbation theory prediction. The dotted line shows an empirical simulation of the inelastic omega $\pi$ contribution obtained by multiplying the inverse amplitude result by the factor $1+0.15 s / s_{\omega}$.

This failure should not be considered a failure of chiral perturbative techniques per se - just that as one approaches higher energy the importance of two-loop $\left(\mathcal{O}\left(p^{6}\right)\right)$ and higher terms become important. Although for simple processes such two loop studies have been performed, the number of $p^{6}$ counterterms is well over a hundred and a general chiral analysis at two loop level is not feasible. Nevertheless things are certainly not hopeless, and in the closing chapter of these lectures we present some approaches to extend the validity of chiral predictions to higher energy.

More relevant at this point is to stay near threshold and ask if chiral pertubation methods are predictive. Can they be used as a test of QCD, for example? The answer is definitely yes! We do not have time in these lectures to give a detailed presentation of the status of such tests-a simple example will have to suffice 21 We have seen above how the pion charge radius enables the determination of one of the chiral parameters $-L_{9}^{r}$. A second $-L_{10}^{r}$ can be found from measurement of the axial structure constant $-h_{A}$ - in radiative pion decay $-\pi^{+} \rightarrow e^{+} \nu_{e} \gamma$ or $\pi^{+} \rightarrow e^{+} \nu_{e} e^{+} e^{-}$, for which the decay amplitudes can be written

$$
\mathcal{M}_{\pi^{+} \rightarrow e^{+} \nu_{e} \gamma}=-\frac{e G_{F}}{\sqrt{2}} \cos \theta_{1} M_{\mu \nu}(p, q) \varepsilon^{\mu *}(q) \bar{u}\left(p_{\nu}\right) \gamma^{\nu}\left(1+\gamma_{5}\right) v\left(p_{e}\right)
$$




$$
\begin{aligned}
\mathcal{M}_{\pi^{+} \rightarrow e^{+} \nu_{e} e^{+} e^{-}}= & -\frac{e^{2} G_{F}}{\sqrt{2}} \cos \theta_{1} M_{\mu \nu}(p, q) \frac{1}{q^{2}} \\
& \times \bar{u}\left(p_{2}\right) \gamma^{\mu} v\left(p_{1}\right) \bar{u}\left(p_{\nu}\right) \gamma^{\nu}\left(1+\gamma_{5}\right) v\left(p_{e}\right),
\end{aligned}
$$

ans the hadronic component of $M_{\mu \nu}$ has the structure

$$
\begin{aligned}
M_{\mu \nu}(p, q) & =\int d^{4} x e^{i q \cdot x}<0 \mid T\left(J_{\mu}^{\mathrm{em}}(x) J_{\nu}^{1-i 2}(0) \mid \pi(\vec{p})>=\right.\text { Born terms } \\
& -h_{A}\left((p-q)_{\mu} q_{\nu}-g_{\mu \nu} q \cdot(p-q)\right)-r_{A}\left(q_{\mu} q_{\nu}-g_{\mu \nu} q^{2}\right) \\
& +i h_{V} \epsilon_{\mu \nu \alpha \beta} q^{\alpha} p^{\beta}
\end{aligned}
$$

where $h_{A}, r_{A}, h_{V}$ are unknown structure functions. (Note that $r_{A}$ can be measured only via the rare Dalitz decay $\pi^{+} \rightarrow e^{+} \nu_{e} e^{+} e^{-}$.)

We also note that the related amplitude for Compton scattering can be written in the form

$$
\begin{aligned}
-i T_{\mu \nu}\left(p, p^{\prime}, q\right) & =-i \int d^{4} x e^{i q_{1} \cdot x}<\pi^{+}\left(\vec{p}^{\prime}\right) \mid T\left(J_{\mu}^{\mathrm{em}}(x) J_{\nu}^{\mathrm{em}}(0) \mid \pi^{+}(\vec{p})>\right. \\
& =\text { Born terms }+\sigma\left(q_{2 \mu} q_{1 \nu}-g_{\mu \nu} q_{1} \cdot q_{2}\right)+\cdots
\end{aligned}
$$

The $\gamma \pi^{+} \rightarrow \gamma \pi^{+}$reaction is often analyzed in terms of the pion electric and magnetic polarizabilities $\alpha_{E}$ and $\beta_{M}$ which describe the response of the pion to external electric and magnetizing fields 22 In the static limit such fields induce electric and magnetic dipole moments

$$
\vec{p}=4 \pi \alpha_{E} \vec{E}, \quad \vec{\mu}=4 \pi \beta_{M} \vec{H}
$$

which correspond to an interaction energy

$$
U=-\frac{1}{2}\left(4 \pi \alpha_{E} \vec{E}^{2}+4 \pi \beta_{M} \vec{H}^{2}\right)
$$

Use of chiral perturbation theory yields the results

$$
\begin{aligned}
h_{V} & =\frac{N_{c}}{12 \sqrt{2} \pi^{2} F_{\pi}}=0.027 m_{\pi}^{-1}, \quad \frac{h_{A}}{h_{V}}=32 \pi^{2}\left(L_{9}^{r}+L_{10}^{r}\right) \\
\frac{r_{A}}{h_{V}} & =32 \pi^{2}\left[L_{9}^{r}-\frac{1}{192 \pi^{2}}\left(\ln \frac{m_{\pi}^{2}}{\mu^{2}}+1\right)\right], \quad \alpha_{E}+\beta_{M}=0 \\
\alpha_{E} & =\frac{\alpha}{2 m_{\pi}} \sigma=\frac{4 \alpha}{m_{\pi} F_{\pi}^{2}}\left(L_{9}^{r}+L_{10}^{r}\right)
\end{aligned}
$$

Use of the experimental result

$$
\frac{h_{A}}{h_{V}}=0.46 \pm 0.08 \quad \text { gives } \quad L_{10}^{r}\left(\mu=m_{\eta}\right)=-0.0056(3)
$$




\begin{tabular}{cccc}
\hline \hline Reaction & Quantity & Theory & Experiment \\
\hline$\pi^{+} \rightarrow e^{+} \nu_{e} \gamma$ & $h_{V}\left(m_{\pi}^{-1}\right)$ & 0.027 & $0.029 \pm 0.0123$ \\
$\pi^{+} \rightarrow e^{+} \nu_{e} e^{+} e^{-}$ & $r_{V} / h_{V}$ & 2.6 & $2.3 \pm 0 . \mathrm{en}^{23}$ \\
$\gamma \pi^{+} \rightarrow \gamma \pi^{+}$ & $\left(\alpha_{E}+\beta_{M}\right)\left(10^{-4} \mathrm{fm}^{3}\right)$ & 0 & $1.4 \pm 3.24$ \\
& $\alpha_{E}\left(10^{-4} \mathrm{fm}^{3}\right)$ & 2.8 & $6.8 \pm 1.25$ \\
& & & $12 \pm 21 \pm 1.26$ \\
\hline
\end{tabular}

Table 3: Chiral Predictions and data in radiative pion processes.

and once this is determined chiral symmetry makes four predictions among these parameters! As shown in Table 3, three of the four are found to be in good agreement with experiment. The possible exception involves a relation between the charged pion polarizability and the axial structure constant $h_{A}$ measured in radiative pion decay. In this case there exist three conflicting experimental results, one of which agrees and one of which does not agree with the theoretical prediction. It is important to resolve this potential discrepancy, since such chiral predictions are firm ones. There is no way (other than introducing perversely large higher order effects) to bring things into agreement were some large violation of a chiral prediction to be verified, since the only ingredient which goes into such predictions is the (broken) chiral symmetry of QCD itself!

\section{Baryon Chiral Perturbation Theory}

Our discussion of chiral methods given above was limited to the study of the interactions of the pseudoscalar mesons (would-be Goldstone bosons) with leptons and with each other. In the real world, of course, interactions with baryons also take place and it is an important problem to develop a useful predictive scheme based on chiral invariance for such processes. Again much work has been done in this regard 28 but there remain important problems 29 Writing down the lowest order chiral Lagrangian at the $\mathrm{SU}(2)$ level is straightforward-

$$
\mathcal{L}_{\pi N}=\bar{N}\left(i \not D-m_{N}+\frac{g_{A}}{2} \psi \gamma_{5}\right) N
$$

where $g_{A}$ is the usual nucleon axial coupling in the chiral limit, the covariant derivative $D_{\mu}=\partial_{\mu}+\Gamma_{\mu}$ is given by

$$
\Gamma_{\mu}=\frac{1}{2}\left[u^{\dagger}, \partial_{\mu} u\right]-\frac{i}{2} u^{\dagger}\left(V_{\mu}+A_{\mu}\right) u-\frac{i}{2} u\left(V_{\mu}-A_{\mu}\right) u^{\dagger},
$$


and $u_{\mu}$ represents the axial structure

$$
u_{\mu}=i u^{\dagger} \nabla_{\mu} U u^{\dagger}
$$

Expanding to lowest order we find

$$
\begin{aligned}
\mathcal{L}_{\pi N} & =\bar{N}\left(i \not \partial-m_{N}\right) N+g_{A} \bar{N} \gamma^{\mu} \gamma_{5} \frac{1}{2} \vec{\tau} N \cdot\left(\frac{i}{F_{\pi}} \partial_{\mu} \vec{\pi}+2 \vec{A}_{\mu}\right) \\
& -\frac{1}{4 F_{\pi}^{2}} \bar{N} \gamma^{\mu} \vec{\tau} N \cdot \vec{\pi} \times \partial_{\mu} \vec{\pi}+\ldots
\end{aligned}
$$

which yields the Goldberger-Treiman relation, connecting strong and axial couplings of the nucleon system 30

$$
F_{\pi} g_{\pi N N}=m_{N} g_{A}
$$

Using the present best values for these quantities, we find

$$
92.4 \mathrm{MeV} \times 13.05=1206 \mathrm{MeV} \text { vs. } 1189 \mathrm{MeV}=939 \mathrm{MeV} \times 1.266
$$

and the agreement to better than two percent strongly confirms the validity of chiral symmetry in the nucleon sector. Actually the Goldberger-Treiman relation is only strictly true at the unphysical point $g_{\pi N N}\left(q^{2}=0\right)$ and one expects about a $1 \%$ discrepancy to exist. An interesting "wrinkle" in this regard is the use of the so-called Dashen-Weinstein relation which uses simple $\mathrm{SU}(3)$ symmetry breaking to predict this discrepancy in terms of corresponding numbers in the strangeness changing sector 31

A second prediction of the lowest order chiral Lagrangian deals with charged pion photoproduction. As emphasized previously, chiral symmetry requires any pion coupling to be in terms of a (co-variant) derivative Hence there exists a $\bar{N} N \pi^{ \pm} \gamma$ contact interaction (the Kroll-Ruderman term 32 which contributes to threshold charged pion photoproduction. Here what is measured is the s-wave or $E_{0+}$ multipole, defined via

$$
\mathrm{Amp}=4 \pi(1+\mu) E_{0+} \vec{\sigma} \cdot \hat{\epsilon}+\ldots
$$

where $\mu=m_{\pi} / M$. In addition to the Kroll-Ruderman piece there exists, at the two derivative level, a second contact term which arises from

$$
\mathcal{L}_{\pi \gamma N N}^{(2)}=\frac{e g_{A}}{8 M F_{\pi}} v \cdot q P_{+}\left[\left(1+\tau_{3}\right) \not A^{\perp}, \gamma_{5} \tau^{a}\right] P_{+}=\frac{e g_{A}}{2 M F_{\pi}} S \cdot \epsilon v \cdot q\left(\tau^{a}+\delta^{a 3}\right)
$$

Adding these two contributions yields the resul 33

$$
\begin{aligned}
E_{0+} & = \pm \frac{1}{4 \pi(1+\mu)} \frac{e g_{A}}{\sqrt{2} F_{\pi}}\left(1 \mp \frac{\mu}{2}\right)=\frac{e g_{A}}{4 \sqrt{2} F_{\pi}}\left(\begin{array}{cc}
1-\frac{3}{2} \mu & \pi^{+} \\
-1+\frac{1}{2} \mu & \pi^{-}
\end{array}\right) \\
& =\left\{\begin{array}{l}
+26.3 \times 10^{-3} / m_{\pi} \pi^{+} n \\
-31.3 \times 10^{-3} / m_{\pi} \pi^{-} p
\end{array}\right.
\end{aligned}
$$




$$
\begin{array}{cl}
E_{0+}^{\pi^{+} n} & (+27.9 \pm 0.5) \times 10^{-3} / m_{\pi}, 3 \\
& (+28.8 \pm 0.7) \times 10^{-3} / m_{\pi} \\
& (+27.6 \pm 0.3) \times 10^{-3} / m_{\pi} 3 \\
E_{0+}^{\pi^{-} p} & (-31.4 \pm 1.3) \times 10^{-3} / m_{\pi} 3 \\
& (-32.2 \pm 1.2) \times 10^{-3} / m_{\pi} \\
& (-31.5 \pm 0.8) \times 10^{-3} / m_{\pi}
\end{array}
$$

Table 4: Experimental values for threshold pion photoproduction multipoles.

and the numerical predictions are found to be in excellent agreement with the present experimental results, as seen in the Table 4.

\subsection{Heavy Baryon Methods}

Extension to SU(3) gives additional successful predictions - the linear GellMann-Okubo relation as well as the generalized Goldbeger-Treiman relation. However, difficulties arise when one attempts to include higher order corrections to this formalism. The difference from the Goldstone case is that there now exist two dimensionful parameters $-m_{N}$ and $F_{\pi}$-in the problem rather than one $-F_{\pi}$. Thus loop effects can be of order $\left(m_{N} / 4 \pi F_{\pi}\right)^{2} \sim 1$ and we no longer have a reliable perturbative scheme. A consistent power counting mechanism can be constructed provided that we eliminate the nucleon mass

from the leading order Lagrangian. This is done by considering the nucleon to be very heavy. Then we can write its four-momentum a 39

$$
p_{\mu}=M v_{\mu}+k_{\mu}
$$

where $v_{\mu}$ is the four-velocity and satisfies $v^{2}=1$, while $k_{\mu}$ is a small off-shell momentum, with $v \cdot k<<M$. One can construct eigenstates of the projection operators $P_{ \pm}=\frac{1}{2}(1 \pm \psi)$, which in the rest frame project out upper, lower components of the Dirac wavefunction, so tha 40

$$
\psi=e^{-i M v \cdot x}\left(H_{v}+h_{v}\right)
$$

where

$$
H_{v}=P_{+} \psi, \quad h_{v}=P_{-} \psi
$$

The effective Lagrangian can then be written in terms of $N, h$ as

$$
\mathcal{L}_{\pi N}=\bar{H}_{v} \mathcal{A} H_{v}+\bar{h}_{v} \mathcal{B} H_{v}+\bar{H}_{v} \gamma_{0} \mathcal{B}^{\dagger} \gamma_{0} h_{v}-\bar{h}_{v} \mathcal{C} h_{v}
$$


where the operators $\mathcal{A}, \mathcal{B}, \mathcal{C}$ have the low energy expansions

$$
\begin{aligned}
\mathcal{A} & =i v \cdot D+g_{A} u \cdot S+\ldots \\
\mathcal{B} & =i \not D^{\perp}-\frac{1}{2} g_{A} v \cdot u \gamma_{5}+\ldots \\
\mathcal{C} & =2 M+i v \cdot D+g_{A} u \cdot S+\ldots
\end{aligned}
$$

Here $D_{\mu}^{\perp}=\left(g_{\mu \nu}-v_{\mu} v_{\nu}\right) D^{\nu}$ is the transverse component of the covariant derivative and $S_{\mu}=\frac{i}{2} \gamma_{5} \sigma_{\mu \nu} v^{\nu}$ is the Pauli-Lubanski spin vector and satisfies the relations

$$
S \cdot v=0, \quad S^{2}=-\frac{3}{4}, \quad\left\{S_{\mu}, S_{\nu}\right\}=\frac{1}{2}\left(v_{\mu} v_{\nu}-g_{\mu \nu}\right), \quad\left[S_{\mu}, S_{\nu}\right]=i \epsilon_{\mu \nu \alpha \beta} v^{\alpha} S^{\beta}
$$

We see that the two components $\mathrm{H}, \mathrm{h}$ are coupled in this expression for the effective action. However, the system may be diagonalized by use of the field transformation

$$
h^{\prime}=h-\mathcal{C}^{-1} \mathcal{B H}
$$

in which case the Langrangian becomes

$$
\mathcal{L}_{\pi N}=\bar{H}_{v}\left(\mathcal{A}+\left(\gamma_{0} \mathcal{B}^{\dagger} \gamma_{0}\right) \mathcal{C}^{-1} \mathcal{B}\right) H_{v}-\bar{h}_{v}^{\prime} \mathcal{C} h_{v}^{\prime}
$$

The piece of the Lagrangian involving $H$ only contains the mass in the operator $\mathcal{C}^{-1}$ and is the effective Lagrangian that we desire. The remaining piece involving $h_{v}^{\prime}$ can be thrown away, as it does not couple to the $H_{v}$ physics. (In path integral language we simply integrate out this component yielding an uninteresting overall constant.) Of course, when loops are calculated a set of counterterms will be required and these are given at leading (two-derivative) order by

$$
\begin{aligned}
\mathcal{A}^{(2)} & =\frac{M}{F_{\pi}^{2}}\left(c_{1} \operatorname{Tr} \chi_{+}+c_{2}(v \cdot u)^{2}+c_{3} u \cdot u+c_{4}\left[S^{\mu}, s^{\nu}\right] u_{\mu} u_{\nu}\right. \\
& \left.+c_{5}\left(\chi_{+}-\operatorname{Tr} \chi_{+}\right)-\frac{i}{4 M}\left[S^{\mu}, S^{\nu}\right]\left(\left(1+c_{6}\right) F_{\mu \nu}^{+}+c_{7} \operatorname{Tr} f_{\mu \nu}^{+}\right)\right) \\
\mathcal{B}^{(2)} & =\frac{M}{F_{\pi}^{2}}\left(\left(-\frac{c_{2}}{4} i\left[u^{\mu}, u^{\nu}\right]+c_{6} f_{+}^{\mu \nu}+c_{7} \operatorname{Tr} f_{+}^{\mu \nu}\right) \sigma_{\mu \nu}\right. \\
& \left.-\frac{c_{4}}{2} v_{\mu} \gamma_{\nu} \operatorname{Tr} u^{\mu} u^{\nu}\right) \\
\mathcal{C}^{(2)} & =-\frac{M}{F_{\pi}^{2}}\left(c_{1} \operatorname{Tr} \chi_{+}+\left(-\frac{c_{2}}{4} i\left[u^{\mu}, u^{\nu}\right]+c_{6} f_{+}^{\mu \nu}+c_{7} \operatorname{tr} F_{+}^{\mu \nu}\right) \sigma_{\mu \nu}\right. \\
& \left.-\frac{c_{3}}{4} \operatorname{Tr} u^{\mu} u_{\nu}-\left(\frac{c_{4}}{2}+M c_{5}\right) v_{\mu} v_{\nu} \operatorname{Tr} u^{\mu} u^{\nu}\right)
\end{aligned}
$$


Expanding $\mathcal{C}^{-1}$ and the other terms in terms of a power series in $1 / M$ leads to an effective heavy nucleon Lagrangian of the form (to $\mathcal{O}\left(q^{3}\right)$ )

$$
\begin{aligned}
\mathcal{L}_{\pi N} & =\bar{H}_{v}\left\{\mathcal{A}^{(1)}+\mathcal{A}^{(2)}+\mathcal{A}^{(3)}+\left(\gamma_{0} \mathcal{B}^{(1) \dagger} \gamma_{0}\right) \frac{1}{2 M} \mathcal{B}^{(1)}\right. \\
& +\frac{\left(\gamma_{0} \mathcal{B}^{(1) \dagger} \gamma_{0}\right) \mathcal{B}^{(2)}+\left(\gamma_{0} \mathcal{B}^{(2) \dagger} \gamma_{0}\right) \mathcal{B}^{(1)}}{2 M} \\
& \left.-\left(\gamma_{0} \mathcal{B}^{(1) \dagger} \gamma_{0}\right) \frac{i(v \cdot D)+g_{A}(u \cdot S)}{(2 M)^{2}} \mathcal{B}^{(1)}\right\} H_{v}+\mathcal{O}\left(q^{4}\right)
\end{aligned}
$$

A set of Feynman rules can now be written down and a consistent pgwer counting scheme developed, as shown by Meissner and his collaborators 29

\subsection{Applications}

As an example of the use of this formalism, called heavy baryon chiral perturbation theory $(\mathrm{HB} \chi \mathrm{pt})$ consider the nucleon-photon interaction. To lowest (one derivative) order we have from $\mathcal{A}^{(1)}$

$$
\mathcal{L}_{\gamma N N}^{(1)}=i e \bar{N} \frac{1}{2}\left(1+\tau_{3}\right) \epsilon \cdot v N
$$

while at two-derivative level we find

$\mathcal{L}_{\gamma N N}^{(2)}=\bar{N}\left\{\frac{e}{4 M}\left(1+\tau_{3}\right) \epsilon \cdot\left(p_{1}+p_{2}\right)+\frac{i e}{2 M}[S \cdot \epsilon, S \cdot k]\left(1+\kappa_{S}+\tau_{3}\left(1+\kappa_{V}\right)\right\} N\right.$

where we have made the identifications $c_{6}=\kappa_{V}, \quad c_{7}=\frac{1}{2}\left(\kappa_{S}-\kappa_{V}\right)$. We can now reproduce the low energy theorems for Compton scattering. Consider the case of the proton. At the two derivative level, we have the tree level prediction

$$
\left.\left(\gamma_{0} \mathcal{B}^{(1) \dagger} \gamma_{0}\right) \frac{1}{2 M} \mathcal{B}^{(1)}\right|_{\gamma p p}=\frac{e^{2}}{2 M} \vec{A}_{\perp}^{2}
$$

which yields the familiar Thomson amplitude

$$
\operatorname{Amp}_{\gamma p p}=-\frac{e^{2}}{M} \hat{\epsilon}^{\prime} \cdot \hat{\epsilon}
$$

On the other hand at order $q^{3}$ we find a contribution from Born diagrams with two-derivative terms at each vertex, yielding

$$
\begin{aligned}
\operatorname{Amp}_{\gamma p p} & =\left(\frac{e}{M}^{2}\right) \frac{1}{\omega} \bar{p}\left[\left(\hat{\epsilon}^{\prime} \cdot \vec{k} \vec{S} \cdot \hat{\epsilon} \times \vec{k}-\hat{\epsilon} \cdot \vec{k}^{\prime} \vec{S} \cdot \hat{\epsilon}^{\prime} \times \vec{k}^{\prime}\right)\left(1+\kappa_{p}\right)\right. \\
& \left.+i \vec{S} \cdot(\hat{\epsilon} \times \vec{k}) \times\left(\hat{\epsilon}^{\prime} \times \vec{k}^{\prime}\right)\left(1+\kappa_{p}\right)^{2}\right]
\end{aligned}
$$




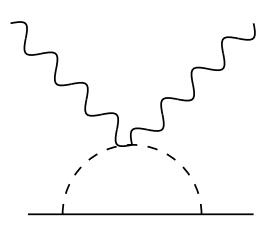

(a)

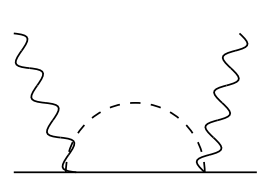

(b)

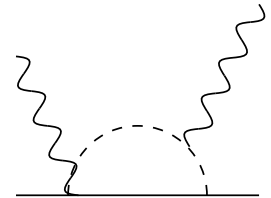

(c)

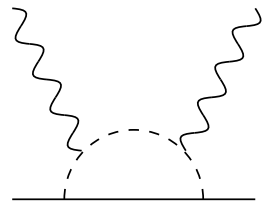

(d)

Figure 7: Loop diagrams for Compton scattering. Figures b,c,d must, of course, be augmented by appropriate cross diagrams.

The full result must also include contact terms at order $q^{3}$ from the last piece of Eq. 148

$$
-e P_{+} \not A^{\perp} \frac{i v \cdot D}{(2 M)^{2}} e \not A^{\perp} P_{+}=-\frac{e^{2}}{2 M^{2}} \vec{S} \cdot \vec{A} \times \dot{\vec{A}}
$$

and from the third

$$
\frac{1}{2 M} P_{+}\left\{e \not A^{\perp}, \kappa_{p} \sigma_{\mu \nu} F^{\mu \nu}\right\} P_{+}=\kappa_{p} \frac{e^{2}}{M^{2}} \vec{S} \cdot \vec{A} \times \dot{\vec{A}}
$$

When added to the Born contributions the result can be expressed in the general form 29

$$
\begin{aligned}
\operatorname{Amp} & =\hat{\epsilon} \cdot \hat{\epsilon}^{\prime} A_{1}+\hat{\epsilon}^{\prime} \cdot \vec{k} \hat{\epsilon} \cdot \vec{k}^{\prime} A_{2}+i \vec{\sigma} \cdot\left(\hat{\epsilon}^{\prime} \times \hat{\epsilon}\right) A_{3} \\
& +i \vec{\sigma} \cdot\left(\vec{k}^{\prime} \times \vec{k}\right) \hat{\epsilon}^{\prime} \cdot \hat{\epsilon} A_{4}+i \vec{\sigma} \cdot\left[\left(\hat{\epsilon}^{\prime} \times \vec{k}\right) \hat{\epsilon} \cdot \vec{k}^{\prime}-\left(\hat{\epsilon} \times \vec{k}^{\prime}\right) \hat{\epsilon}^{\prime} \cdot \vec{k}\right] A_{5} \\
& +i \vec{\sigma} \cdot\left[\left(\hat{\epsilon}^{\prime} \times \vec{k}^{\prime}\right) \hat{\epsilon} \cdot \vec{k}^{\prime}-(\hat{\epsilon} \times \vec{k}) \hat{\epsilon}^{\prime} \cdot \vec{k}\right] A_{6}
\end{aligned}
$$

with

$$
\begin{aligned}
& A_{1}=-\frac{e^{2}}{M}, \quad A_{2}=\frac{1}{M^{2} \omega}, \quad A_{3}=\frac{e^{2} \omega}{2 M^{2}}\left(1+2 \kappa-(1+\kappa)^{2} \hat{k} \cdot \hat{k}^{\prime}\right) \\
& A_{4}=-A_{5}=-\frac{e^{2}(1+\kappa)^{2}}{2 M^{2} \omega},
\end{aligned}
$$

which agrees with the usual result derived in this order via Low's theorem 41

${ }^{c}$ Here we have used the identity

$\vec{\sigma} \cdot\left(\hat{\epsilon}^{\prime} \times \vec{k}^{\prime}\right) \times(\hat{\epsilon} \times \vec{k})=\vec{\sigma} \cdot\left(\vec{k}^{\prime} \times \vec{k}\right) \hat{\epsilon} \cdot \hat{\epsilon}^{\prime}+\vec{\sigma} \cdot\left(\hat{\epsilon}^{\prime} \times \hat{\epsilon}\right) \vec{k}^{\prime} \cdot \vec{k}+\vec{\sigma} \cdot\left(\hat{\epsilon} \times \vec{k}^{\prime}\right) \hat{\epsilon}^{\prime} \cdot \vec{k}-\vec{\sigma} \cdot\left(\hat{\epsilon}^{\prime} \times \vec{k}\right) \hat{\epsilon} \cdot \vec{k}^{\prime}$ 
A full calculation at order $q^{3}$ must also, of course, include loop contributions. Using the lowest order (one-derivative) pion-nucleon interactions

$$
\begin{aligned}
\mathcal{L}_{\pi N N} & =\frac{g_{A}}{F_{\pi}} \bar{N} \tau^{a} S \cdot q N \\
\mathcal{L}_{\pi \pi N N} & =\frac{1}{4 F_{\pi}^{2}} v \cdot\left(q_{1}+q_{2}\right) \epsilon^{a b c} \bar{N} \tau_{c} N \\
\mathcal{L}_{\gamma \pi N N} & =\frac{i e g_{A}}{F_{\pi}} \epsilon^{a 3 b} \bar{N} \epsilon \cdot S \tau_{b} N
\end{aligned}
$$

these can be calculated using the diagrams shown in Figure 7. Of course, from Eq. 148 the propagator for the nucleon must have the form $1 / i v \cdot k$ where $\mathrm{k}$ is the off-shell momentum. Thus, for example, the seagull diagram, Figure 7a, is of the form

$$
\operatorname{Amp}=4 e^{2}\left(\frac{g_{A}}{F_{\pi}}\right)^{2} \hat{\epsilon} \cdot \hat{\epsilon}^{\prime} \int \frac{d^{4} k}{(2 \pi)^{4}} \frac{S \cdot k S \cdot k}{v \cdot k\left(k^{2}-m_{\pi}^{2}\right)\left(\left(k+q_{1}-q_{2}\right)^{2}-m_{\pi}^{2}\right)}
$$

Since there are no additional counterterms at this order $q^{3}$, the sum of loop diagrams must be finite and yields, to lowest order in energy and after considerable calculation

$$
\begin{aligned}
& A_{1}^{\text {loop }}=\xi\left(\frac{11 \omega^{2}}{24 m_{\pi}}+\frac{t}{48 m_{\pi}}\right), \quad A_{2}^{\text {loop }}=\xi\left(\frac{1}{24 m_{\pi}}\right), \quad A_{3}^{\text {loop }}=\xi\left(\frac{\omega t}{\pi m_{\pi}^{2}}+\frac{\omega^{3}}{3 \pi m_{\pi}^{2}}\right) \\
& A_{4}^{\text {loop }}=\xi\left(\frac{\omega}{6 \pi m_{\pi}^{2}}\right), \quad A_{5}^{\text {loop }}=-A_{6}^{\text {loop }}=-\xi\left(\frac{13 \omega}{12 \pi m_{\pi}^{2}}\right),
\end{aligned}
$$

and $\xi=g_{A}^{2} / 8 \pi F_{\pi}^{2}$.

The experimental implications of these results may be seen by first considering the case of an unpolarized proton target. Writing

$$
\mathrm{Amp}_{\text {unpol }}=\left\{\hat{\epsilon} \cdot \hat{\epsilon}^{\prime}\left(-\frac{e^{2}}{M}+4 \pi \alpha_{E} \omega^{2}\right)+(\hat{\epsilon} \times \vec{k}) \cdot\left(\hat{\epsilon}^{\prime} \times \vec{k}^{\prime}\right) 4 \pi \beta_{M}\right\}
$$

where $\alpha_{E}, \beta_{M}$ are the proton electric and magnetic polarizabilities, we identify the one loop chiral prediction 42

$$
\alpha_{E}^{\text {theo }}=10 \beta_{M}^{\text {theo }}=\frac{5 e^{2} g_{A}^{2}}{384 \pi^{2} F_{\pi}^{2} m_{\pi}}=12.2 \times 10^{-4} \mathrm{fm}^{3}
$$

which are in reasonable agreement with the recently measured value 43

$$
\alpha_{E}^{\text {exp }}=(12.1 \pm 0.8 \pm 0.5) \times 10^{-4} \mathrm{fm}^{3}, \quad \beta_{M}^{e x p}=(2.1 \mp 0.8 \mp 0.5) \times 10^{-4} \mathrm{fm}^{3}
$$


For the case of spin-dependent forward scattering, we find, in general

$$
\frac{1}{4 \pi} \operatorname{Amp}=f_{1}\left(\omega^{2}\right) \hat{\epsilon} \cdot \hat{\epsilon}^{\prime}+i \omega f_{2}\left(\omega^{2}\right) \vec{\sigma} \cdot \hat{\epsilon}^{\prime} \times \hat{\epsilon}
$$

with

$$
\begin{aligned}
& f_{1}\left(\omega^{2}\right)=-\frac{e^{2}}{4 \pi M}+\left(\alpha_{E}+\beta_{M}\right) \omega^{2}+\mathcal{O}\left(\omega^{4}\right) \\
& f_{2}\left(\omega^{2}\right)=-\frac{e^{2} \kappa_{p}^{2}}{8 \pi^{2} M^{2}}+\gamma_{S} \omega^{2}+\mathcal{O}\left(\omega^{4}\right)
\end{aligned}
$$

where $\gamma_{S}$ is a sort of "spin-polarizability" and is related to the classical Faraday effect. Assuming that the amplitudes $f_{1}, f_{2}$ obey once-subtracted and unsubtracted dispersion relations respectively we find the sum rules

$$
\begin{aligned}
\alpha_{E}+\beta_{M} & =\frac{1}{4 \pi^{2}} \int_{\omega_{0}}^{\infty} \frac{d \omega}{\omega^{2}}\left(\sigma_{+}(\omega)+\sigma_{-}(\omega)\right) \\
\frac{\pi e^{2} \kappa_{p}^{2}}{2 M^{2}} & =\int_{\omega_{0}}^{\infty} \frac{d \omega}{\omega}\left[\sigma_{+}(\omega)-\sigma_{-}(\omega)\right] \\
\gamma_{S} & =\frac{1}{4 \pi^{2}} \int_{\omega_{0}}^{\infty} \frac{d \omega}{\omega^{3}}\left[\sigma_{+}(\omega)-\sigma_{-}(\omega)\right]
\end{aligned}
$$

where here $\sigma_{ \pm}(\omega)$ denote the photoabsorption cross sections for scattering circularly polarized photons on polarized nucleons invovling total $\gamma N$ helicity $3 / 2$ and $1 / 2$ respectively. Here the first is the well-known Baldin sum rule for the sum of the electric and magnetic polarizabilities, while the second is the equally familiar Drell-Hearn-Gerasimov sum rule 4 The third is less well known, but follows from that of DHG and offeres a new check of the chiral predictions.

Another venue where there has been a great deal of work is that of neutral pion photoproduction, for which the one-derivative contribution vanishes. In this case the leading contribution arises from the two derivative term given in Eq. 148, augmented by the three derivative contribution from Born diagrams. The net result is

$$
\operatorname{Amp}^{(2)}=\frac{e g_{A}}{2 F_{\pi}} \mu \hat{\epsilon} \cdot \vec{\sigma} \times \begin{cases}1 & \pi^{0} p \\ 0 & \pi^{0} n\end{cases}
$$

for the contact term and

$$
\mathrm{Amp}^{(3)}=-\frac{e}{2 M}[S \cdot \epsilon, S \cdot k]\left(1+\kappa_{p}\right) \frac{1}{v \cdot q} \frac{g_{A}}{2 M F_{\pi}} S \cdot(2 p-q) m_{\pi}=-\frac{e g_{A}}{4 F_{\pi}} \mu^{2}\left(1+\kappa_{p}\right) \vec{\sigma} \cdot \vec{\epsilon}
$$




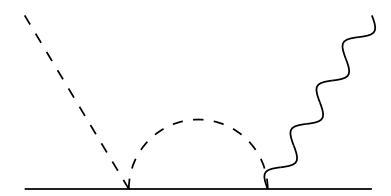

(a)

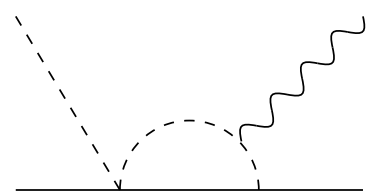

(b)

Figure 8: Diagrams for neutral pion photoproduction. Each should be accompanied by an appropriate cross term.

$$
\begin{array}{ccc} 
& \text { theory } & \text { expt. } \\
E_{0+}\left(\pi^{0} p\right)\left(\times 10^{-3} / m_{\pi}\right) & -1.2 & -1.31 \pm 0.0 \$ 46 \\
& & -1.32 \pm 0.1] \\
E_{0+}\left(\pi^{0} n\right)\left(\times 10^{-3} / m_{\pi}\right) & 2.1 & 1.9 \pm 0.48 \\
P_{1} /|\vec{q}|(\pi p)\left(\times \mathrm{GeV}^{-2}\right) & 0.48 & 0.47 \pm 0.0146 \\
& & 0.41 \pm 0.0 .47
\end{array}
$$

Table 5: Threshold parameters for neutral pion photoproduction.

for the pole terms (Note: only the cross term is nonvanishing at threshhold.)

Finally we must append the contribution of the loop contributions which arise from the graphs shown in Figure 8

$$
\operatorname{Amp}^{l o o p}=-\frac{e g_{A} M}{64 \pi F_{\pi}^{2}} \mu^{2} \vec{\sigma} \cdot \vec{\epsilon}
$$

The result is the prediction 45

$$
E_{0+}=\frac{e g_{A}}{8 \pi M} \mu\left\{1-\left[\frac{1}{2}\left(3+\kappa_{p}\right)+\left(\frac{M}{4 F_{\pi}}\right)^{2}\right] \mu+\mathcal{O}\left(\mu^{2}\right)\right\}
$$

However, comparison with experiment is tricky because of the existence of isotopic spin breaking in the pion and nucleon masses, so that there are two thresholds - one for $\pi^{0} p$ and the second for $\pi^{+} n$ - only $7 \mathrm{MeV}$ apart. When the physical masses of the pions are used recent data from both Mainz and from Saskatoon agree with the chiral prediction. However, there are concerns about the convergence of the chiral expansion, which reads $E_{0+}=C(1-1.26+$ $0.59+\ldots)$. There also exist chiral predictions for threshold p-wave amplitudes which are in good agreement with experiment, as shown in Table 5, and for which the convergence is calculated to be rapid. 
Finally exists a chiral symmetry prediction for the reaction $\gamma n \rightarrow \pi^{0} n$

$$
E_{0+}=-\frac{e g_{A}}{8 \pi M} \mu^{2}\left\{\frac{1}{2} \kappa_{n}+\left(\frac{M}{4 F_{\pi}}\right)^{2}\right\}+\ldots=2.13 \times 10^{-3} / m_{\pi}
$$

However, the experimental measurement of such an amplitude involves considerable challenge, and must be accomplished either by use of a deuterium target with the difficult subtraction of the proton contribution and of meson exchange contributions or by use of a ${ }^{3} \mathrm{He}$ target. Neither of these are straightforward although some limited data already exist 4

Other areas wherein chiral predictions can be confronted with experiment include the electric dipole amplitude in electroproduction as well as in weak interactions such as muon capture. However, we do not have the space here to cover this work. We can succinctly summarize the situation by stating that at the present time there exist no significant disagreements with experimental findings, but the predictive power in the baryon sector is only in the near threshold region, due among other things to the feature that the expansion is in terms of $p / m$ rather than $p^{2} / m^{2}$ which occurs in the meson case.

\section{On to Higher Energy: Dispersion Relations}

Above we have seen the power of chiral perturbation theory in addressing near-threshold phenomenology. However, we have also observed its associated weakness - loss of predictive ability in the meson sector once $E, p \geq \sim m_{\rho} / 2$ and in the baryon-meson sector even sooner. In this closing chapter, we try to address the question of whether one can somehow extend the success of $\chi \mathrm{pt}$ to higher energy without losing its model-independent connections to QCD. Strictly speaking the answer is no-as the energy-momentum increases one must go to higher and higher order in the chiral expansion, which means increasing the number of loops and of unknown counterterms. Any other approach must be model dependent at some level and hence use more than simply the (broken) chiral symmetry of QCD as an input. Nevertheless, there are some techniques which keep such model dependence at a minimum. One such procedure is to marry the results of $\chi \mathrm{pt}$ with the strictures of dispersion relations the validity of which, given that they rely only on the causality properties of the theory, are not in question. In the case of the pion form factor studied above in one loop $\chi \mathrm{pt}$ the causality condition asserts that the function $F\left(q^{2}\right)$ is analytic in the entire complex $q^{2}$-plane except for a cut along the real axis which extends from $4 m_{\pi}^{2}<q^{2}<\infty$. Along this cut $F\left(q^{2}\right)$ has an 


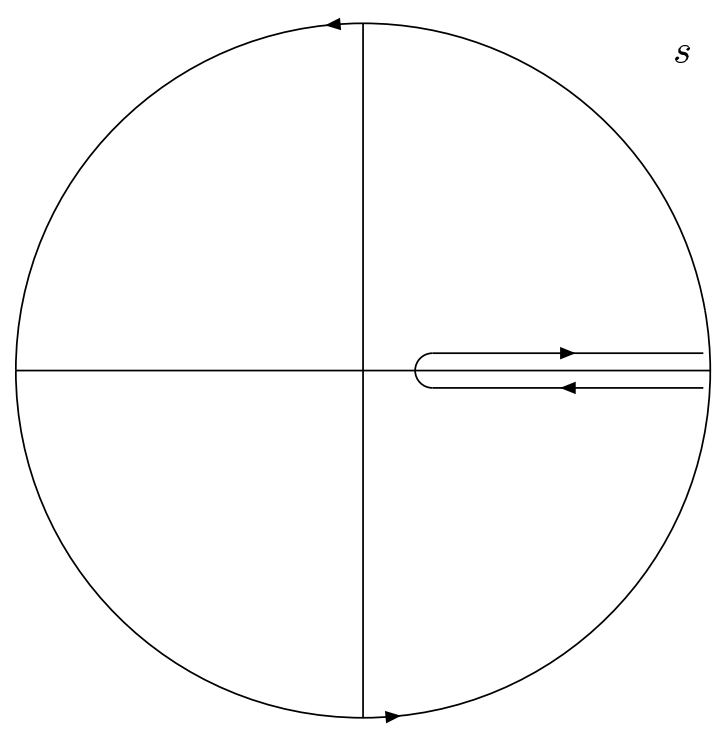

Figure 9: Contour for form factor integration.

imaginary part which is given by the unitarity condition

$$
\begin{aligned}
\left(p_{2}-p_{1}\right)_{\mu} \operatorname{Im} F_{1}\left(q^{2}\right) & =\frac{1}{2} \int \frac{d^{3} k_{1}}{(2 \pi)^{3} 2 E_{1}} \frac{d^{3} k_{2}}{(2 \pi)^{3} 2 E_{2}}(2 \pi)^{4} \delta^{4}\left(p_{1}+p_{2}-k_{1}-k_{2}\right) \\
& \times<\pi \pi|T| \pi \pi>^{*}<\pi \pi\left|j_{\mu}\right| 0>
\end{aligned}
$$

Use of Cauchy's theorem involving the contour as shown in Figure 9 and the assumption that $F(s) \rightarrow 0$ as $s \rightarrow \infty$ then leads to the dispersion relation

$$
F_{1}\left(q^{2}\right)=\frac{1}{\pi} \int_{4 m_{\pi}^{2}}^{\infty} \frac{\operatorname{Im} F_{1}(s) d s}{s-q^{2}-i \epsilon}
$$

If the asymptotic condition is not satisfied one can use various numbers of subtractions in order to guarantee convergence. For example, provided that $F_{1}(s) / s \rightarrow 0$ as $s \rightarrow \infty$ we have

$$
\begin{aligned}
F_{1}\left(q^{2}\right)-F_{1}(0) & =\frac{1}{\pi} \int_{4 m_{\pi}^{2}}^{\infty} \operatorname{Im} F_{1}(s) d s\left(\frac{1}{s-q^{2}-i \epsilon}-\frac{1}{s-i \epsilon}\right) \\
& =\frac{q^{2}}{\pi} \int_{4 m_{\pi}^{2}}^{\infty} \frac{\operatorname{Im} F_{1}(s) d s}{s\left(s-q^{2}-i \epsilon\right)}
\end{aligned}
$$


and further subtractions may be performed if necessary. Even if such subtractions are not required it may be advantageous to utilize them. The point is that in order to get a prediction one requires the value of the imaginary component of the form factor at all values of $s$. However, the more subtractions the less the sensitivity to input from large $s$, where in general the form factor is not as well determined.

In the case of the pion form factor it is convenient to perform two subtractions, whereby

$$
F_{1}\left(q^{2}\right)=F_{1}(0)+q^{2} F_{1}^{\prime}(0)+\frac{q^{4}}{\pi} \int_{4 m_{\pi}^{2}}^{\infty} \frac{\operatorname{Im} F_{1}(s) d s}{s^{2}\left(s-q^{2}-i \epsilon\right)}
$$

Here by current conservation we may set $F_{1}(0)=1$ while from experiment we have $F_{1}^{\prime}(0)=<r_{\pi}^{2}>/ 6=(0.073 \pm 0.002) \mathrm{fm}^{2}$. In order to find $\operatorname{Im} F(s)$ we begin by projecting onto the p-wave $\pi \pi$ channel via

$$
T_{1}^{1}(s)=\frac{1}{64 \pi} \int_{-1}^{1} d(\cos \theta) P_{1}(\cos \theta)<\pi \pi|T| \pi \pi>=\sqrt{\frac{s}{s-4 m_{\pi}^{2}}} e^{i \delta_{1}^{1}} \sin \delta_{1}^{1}
$$

whereby the unitarity condtion Eq. 172 reads

$$
\operatorname{Im} F_{1}(s)=e^{-i \delta_{1}^{1}} \sin \delta_{1}^{1} F_{1}(s)
$$

Of course, the solution of this equation is simply the Fermi-Watson theorem

$$
F_{1}(s)=\left|F_{1}(s)\right| \exp i \delta_{1}^{1}(s)
$$

which states that the phase of the form factor is the p-wave $\pi \pi$ phase shift. Now at lowest order we have the chiral prediction

$$
T_{1}^{1}(s)=\frac{s-4 m_{\pi}^{2}}{96 \pi F_{\pi}^{2}}
$$

and if this is substituted into Eq. 177 along with the lowest order result $F_{1}(s) \approx F_{1}(0)=1$ we find

$$
F_{1}\left(q^{2}\right)=1+\frac{<r_{\pi}^{2}>}{6} q^{2}+\frac{1}{96 \pi^{2} F_{\pi}^{2}}\left(\left(q^{2}-4 m_{\pi}^{2}\right) H\left(q^{2}\right)+\frac{2}{3} q^{2}\right)
$$

where we have used the integral

$$
\int_{4 m_{\pi}^{2}}^{\infty} \frac{d s}{s^{2}} \sqrt{\frac{s-4 m_{\pi}^{2}}{s}}\left(\frac{a+b s}{s-q^{2}-i \epsilon}\right)=\frac{a+b q^{2}}{q^{4}} H\left(q^{2}\right)-\frac{a}{6 m^{2} q^{2}}
$$


Comparison with the chiral perturbative result-Eq. 120 reveals that the results are identical provided that one identifies

$$
\frac{<r_{\pi}^{2}>}{6}=\frac{2 L_{9}^{r}}{F_{\pi}^{2}}+\frac{1}{96 \pi^{2} F_{\pi}^{2}}\left(1+\ln \frac{m_{\pi}^{2}}{\mu^{2}}\right)
$$

In this form then we see that the chiral perturbative predictions are simply a result of use of the lowest order chiral forms in the input to the dispersion integral, while a real world calculation would utilize experimental data. In this context it is clear that the renormalized counterterms simply play the role of subtraction constants.

So far our dispersive calculation has simply reproduced the chiral perturbative results. How can one do better? Clearly hy using experimental data rather then the simple lowest order chiral forms 49 For example, if we divide Eq. 175 by $q^{2}$ and then take the limit as $q^{2} \rightarrow \infty$ we find a sum rule for $L_{9}^{r}(\mu)$

$$
L_{9}^{r}(\mu)=\frac{F_{\pi}^{2}}{6 \pi} \int_{4 m_{\pi}^{2}}^{\infty} \frac{d s}{s^{2}} \operatorname{Im} F_{1}(s)-\frac{1}{192 \pi^{2}}\left(1+\ln \frac{m_{\pi}^{2}}{\mu^{2}}\right)
$$

which can be evaluated from experiment. Actual data on $\operatorname{Im} F(s)$ are available only from the $\pi \pi$ threshold up to the middle of the resonance region so other methods must be found in order to generate the high energy component required in order to perform the dispersive integral. For very high energies we can use the prediction of perturbative QCD

$$
F_{1}(s) \longrightarrow-\frac{64 \pi^{2}}{9} \frac{F_{\pi}^{2}}{s \ln \frac{-s}{\Lambda^{2}}}
$$

which corresponds to the asymptotic form

$$
\operatorname{Im} F_{1}(s) \longrightarrow-\frac{64 \pi^{3}}{9} \frac{F_{\pi}^{2}}{s \ln ^{2} \frac{s}{\Lambda^{2}}}
$$

Knowing the low and high energy forms of the function, we can generate a smooth matching-which joins them in the intermediate energy region, as shown in Figure 149. Obviously the most striking part of this function is the strong $\rho$ peak, but note also the long negative tail at high energy. That the high energy component must be negative is easy to see from the fact that Eq. 185 guarantees that the dispersion integral converges even without any subtractions. Then, taking the $q^{2} \rightarrow \infty$ limit produces two additional sum rules

$$
1=\frac{1}{\pi} \int_{4 m_{\pi}^{2}}^{\infty} \frac{d s}{s} \operatorname{ImF}_{1}(\mathrm{~s})
$$




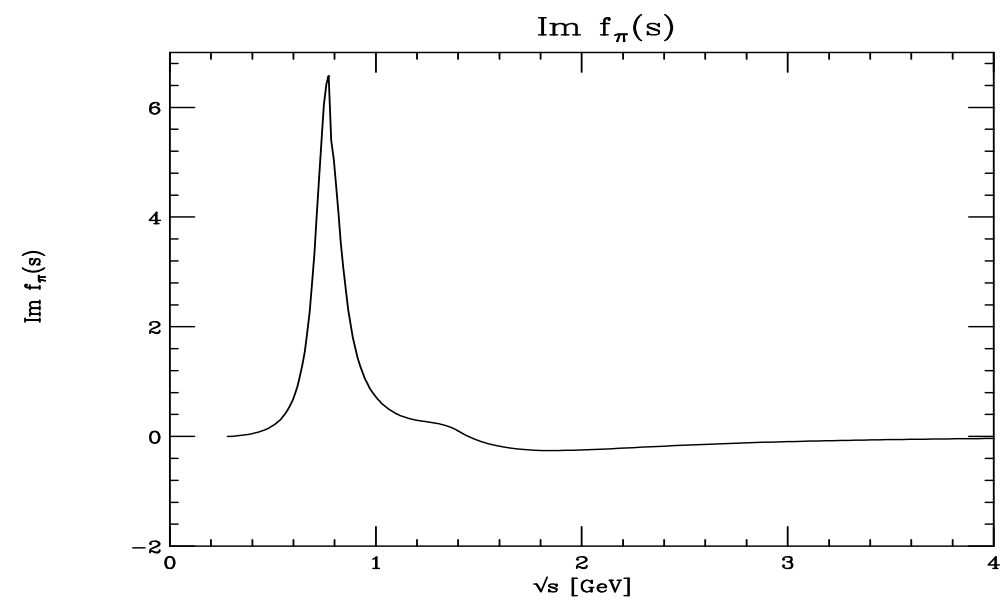

Figure 10: Input data for the pion form factor dispersion relation.

$$
0=\frac{1}{\pi} \int_{4 m_{\pi}^{2}}^{\infty} d s \operatorname{Im} F_{1}(s)
$$

In particular the latter sum rule requires that the large positive contribution generated by integrating over the rho peak be cancelled by a significant negative result from higher energy. Now in fact both sum rules are satisfied by the spectral function shown in Figure 10, as they were used in its construction and are responsible for the small bump to the right of the rho tail. Having generated a consistent form for $\operatorname{Im} F(s)$ we can now use Eq. 183 in order to generate a prediction for $L_{9}^{r}$. We find

$$
\left.L_{9}^{r}\left(m_{\eta}\right)\right|^{\text {disp.rel. }}=0.0074 \quad \text { compared to }\left.\quad L_{9}^{r}\left(m_{\eta}\right)\right|^{\text {expt. }}=0.0071(3)
$$

obtained from analysis of low energy phenomenology (specifically the pion charge radius). Obviously there is good agreement here, confirming the validity of the dispersive approach (and therefore causality!)

However, while we have gained a new understanding of the chiral perturbative results we have not yet succeeded in extending their validity to higher energy. Indeed, Figure 6 shows that, while the form factor $F\left(q^{2}\right)$ matches onto the experimental result at low $q^{2}$, it is strongly at variance by the time $q^{2} \approx 400 \mathrm{MeV}^{2}$ due to the stron rise of the rho. In fact the prominance of the rho peak suggests an oft-used approximation - replacement of the spectral 
density by a simple delta function

$$
\operatorname{Im} F_{1}(s)=\pi m_{\rho}^{2} \delta\left(s-m_{\rho}^{2}\right)
$$

This drastic approximation - called "vector dominance" - does not satisfy the barely convergent sum rule Eq. 186a but explicitly satisfies the better damped Eq. 186 b. With respect to that for $L_{9}$ we find

$$
<r_{\pi}^{2}>=6 m_{\rho}^{2} \simeq 0.40 \mathrm{fm}^{2}
$$

in reasonable agreement with experiment.

An approach which does allow us to move to higher energy is the "inverse amplitude method," wherein one looks not at the form factor but rather at its inverse 50 The unitarity condition in this case reads

$$
\operatorname{Im} \frac{1}{F_{1}(s)}=-\frac{e^{i \delta_{1}^{1}(s)} \sin \delta_{1}^{1}(s)}{F_{1}(s)}=-\sqrt{\frac{s-4 m_{\pi}^{2}}{s}} \frac{T_{1}^{1}(s)}{F_{1}(s)}
$$

and a doubly subtracted dispersion relation reads

$$
\frac{1}{F_{1}\left(q^{2}\right)}=1-\frac{<r_{\pi}^{2}>}{6} q^{2}-\frac{1}{\pi} \int_{4 m_{\pi}^{2}}^{\infty} \frac{d s}{s^{2}\left(s-q^{2}-i \epsilon\right)} \sqrt{\frac{s-4 m_{\pi}^{2}}{s}} \frac{T_{1}^{1}(s)}{F_{1}(s)}
$$

Using the leading chiral forms, the integration can be performed using Eq. 181 and yields

$$
F_{1}\left(q^{2}\right)=\frac{1}{1-\frac{<r_{\pi}^{2}>}{6} q^{2}-\frac{1}{96 \pi^{2} F_{\pi}^{2}}\left(\left(q^{2}-4 m_{\pi}^{2}\right) H\left(q^{2}\right)+\frac{2}{3} q^{2}\right)}
$$

Expanding in $q^{2}$ we observe that this form is consistent with the $\mathcal{O}\left(p^{4}\right)$ chiral result and represents summation of the Lippman-Scwinger equation in terms of a geometric series. (Sometimes this is also called the Pade $(1,1)$ approximant form 50) Phenomenologically it has the right stuff. Indeed, as shown in Figure 6 , the rho resonance arises very naturally as a pole as $s \rightarrow m_{\rho}^{2}$ and the phase shift, given by $\delta_{1}^{1}=\arg F(s)$ is in reasonable agreement with experiment. We stress that it is not a simple result of chiral symmetry - rather it is a chirally motivated form which reduces to the chiral result at low energy.

It is interesting to note that there is an exact solution to the problem of finding a function whose phase is $\delta_{1}^{1}$ along the real axis from $4 m_{\pi}^{2}<s<\infty$. This is the Omnes solution and has the general forn 52

$$
F_{1}^{\text {Omnes }}\left(q^{2}\right)=P\left(q^{2}\right) \exp \frac{q^{2}}{\pi} \int_{4 m_{\pi}^{2}}^{\infty} \frac{d s}{s} \frac{\delta_{1}^{1}(s)}{s-q^{2}-i \epsilon}
$$


Here $P(s)$ is an arbitrary polynomial which for our case is set equal to unity. Using experimental $\pi \pi$ phase shifts one can construct the function $F_{0}\left(q^{2}\right)$ and compare with experiment and with the inverse amplitude form. Agreement with experiment is basically quite good. Note that the Omnes solution assumes the validity of the Fermi-Watson theorem along the entire real axis and hence ignores the substantial inelasticity which arises above $\sim 1.2 \mathrm{GeV}$. Thus this form should presumably only be trusted up to energies where such inelasticity takes over.

One can in similar fashion construct a form for the partial wave scattering amplitude which is unitary

$$
\operatorname{Im} \frac{1}{T_{1}^{1}(s)}=-i \sqrt{\frac{s-4 m_{\pi}^{2}}{s}}
$$

and agrees with the $\pi \pi$ phase shifts found from the form factor as

$$
T_{1}^{1}(s)=\frac{1}{96 \pi^{2} F_{\pi}^{2}} \frac{\left(s-4 m_{\pi}^{2}\right)}{1-\frac{\left\langle r_{\pi}^{2}>\right.}{6} s-\frac{1}{96 \pi^{2} F_{\pi}^{2}}\left(\left(s-4 m_{\pi}^{2}\right) H(s)+\frac{2}{3} s\right)}
$$

In fact, such a form was written down long before the development of chiral perturbative techniques as a simple unitary generalization of the lowest order (Weinberg) scattering amplitude 33 It is sometimes called the N/D form, where $\mathrm{D}$ is the inverse form factor and satisfies the Fermi-Watson theorm along the right-hand cut while $\mathrm{N}$ is the lowest order chiral amplitude and is analytic along this discontinuity. Of course, Eq. 195 violates crossing symmetry in that it does not have the proper discontinuity along the left-hand (u-channel) cut, but we may hope that this does not matter along the physical (right-hand) cut as it is far away.

We see then that by the use of chiral-based methods, one can construct analytic forms for observables which satisfy the strictures of unitarity exactly (not perturbatively as in the case of chiral perturbation theory itself) and which reduce to the forms demanded by chiral symmetry at low energy. They are admittedly no longer model-independent, nor do they satisfy all the strictures of field theory (such as crossing symmetry). However, any reasonable model must assume a similar form and they can be used with some confidence to construct a successful phenomenology.

\section{Closing Comments}

In the preceding lectures we have covered a lot of ground - effective field theory, basic chiral models, chiral perturbation theory in the meson and baryon 
sectors, and high energy extensions based upon dispersion theory. Nevertheless the discussion has essentially been at an introductory level and there is lots more to learn for those who are interested. The state of the art both for experiment and for theory can be found in the proceedings of the first two chiral dynamics workshops (which took place at MIT and Mainz respectively) which were published by Springer-Verlag 54 The next such meeting, by the way, will be held this summer at JLab, and you are certainly invited to attend.

Despite all the work which has taken place there is still much to do for those who wish to take up the challenge. This lies in many areas:

i) calculating to yet higher order (two loop) in both the meson and baryon sectors;

ii) making the connection with fundamental theory by calculating the chiral coefficients directly from QCD by lattice or other methods;

iii) development of reasonably model-independent methods to extend chiral results to higher energy;

iv) identifying and performing the experiments which most sensitively probe such theories.

My basic message, which I hope you take with you, is that chiral perturbative methods are an important and vital component of contemporary particle/nuclear physics and that at present they allow perhaps the best way to confront low energy phenomenology with the fundamental QCD Lagrangian which presumably underlies it.

\section{Acknowledgement}

It is a pleasure to acknowledge warm hospitality of the organizers of this meeting. This work was supported in part by the National Science Foundation.

1. See, e.g. A. Manohar, "Effective Field Theories," in Schladming 1966: Perturbative and Nonperturbative Aspects of Quantum Field Theory, hep-ph/9606222; D. Kaplan, "Effective Field Theories," in Proc. 7th Summer School in Nuclear Physics, nucl-th/9506035,; H. Georgi, "Effective Field Theory," in Ann. Rev. Nucl Sci. 43, 209 (1995).

2. J. Gasser and H. Leutwyler, Ann. Phys. (NY) 158, 142 (1984); Nucl. Phys. B250, 465 (1985).

3. For a pedagogical introduction see J.F. Donoghue, E. Golowich and B.R. Holstein, Dynamics of the Standard Model, Cambridge University Press, New York (1992).

4. B.R. Holstein, Am. J. Phys. 67, 422 (1999). 
5. A corresponding classical physics discussion is given in R.P Feynman, R.B. Leighton, and M. Sands, The Feynman Lecures on Physics, Addison-Wesley, Reading, MA, (1963) Vol. I, Ch. 32.

6. H. Euler, Ann. Phys. (Leipzig) 26, 398 (1936); W. Heisenberg and H. Euler, Z. Phys. 98, 714 (1936).

7. See, e.g. J.F. Donoghue, "Dispersion Relations and Effective Field Theory," hep-ph/9607351.

8. H. Weyl, Symmetry, Princeton University Press, Princeton (1964).

9. See, e.g. J.D. Bjorken and S.D. Drell, Relativisitic Quantum Fields, McGraw-Hill, New York (1964).

10. J. Svardiere, Am. J. Phys. 51, 1016 (1983).

11. This example is taken from R. Jackiw, in M.A.B. Beg Menorial Volume, ed. A. ali and P. Hoodbhoy, World Scientific, Singapore (1991).

12. L.R. Mead and J. Godines, Am. J. Phys. 59, 935 (1991).

13. J. Goldstone, Nuovo Cim. 19, 154 (1961); J. Goldstone, A. Salam and S. Weinberg, Phys. Rev. 127, 965 (1962).

14. C.P. Burgess, "Goldstone and Pseudo-Goldstone Bosons in Nuclear, Particle, and Condensed Matter Physics," hep-ph/9808176.

15. M. Gell-Mann, CalTech Rept. CTSL-20 (1961); S. Okubo, Prog. Theo. Phys. 27, 949 (1962).

16. B.R. Holstein, Phys. Lett. B244, 83 (1990).

17. S. Gasiorowicz and D.A. Geffen, Rev. Mod. Phys. 41, 531 (1969).

18. S. Weinberg, Physica A96, 327 (1979).

19. A. Manohar and H. Georgi, Nucl. Phys. B234, 189 (1984); J.F. Donoghue, E. Golowich and B.R. Holstein, Phys. Rev. D30, 587 (1984).

20. E.B. Dally et al., Phys. Rev. Lett. 48, 375 (1982).

21. J.F. Donoghue and B.R. Holstein, Phys. Rev. D40, 2378 (1989).

22. B.R. Holstein, Comm. Nucl. Part. Phys. 19, 221 (1990).

23. Particle Data Group, Phys. Rev. D54, 1 (1996).

24. Yu. M. Antipov et al., Z. Phys. C26, 495 (1985).

25. Yu. M. Antipov et al., Phys. Lett. B121, 445 (1983).

26. T.A. Aibergenov et al., Czech. J. Phys. 36, 948 (1986).

27. D. Babusci et al., Phys. Lett. B277, 158 (1992).

28. J. Gasser, M. Sainio, and A. Svarc, Nucl. Phys. B307, 779 (1988).

29. V. Bernard, N. Kaiser, and U.G. Meissner, Int. J. Mod. Phys. E4, 193 (1995).

30. M. Goldberger and S.B. Treiman, Phys. Rev. 110, 1478 (1958).

31. R. Dashen and M. Weinstein, Phys. Rev. 188, 2330 (1969); B.R. Holstein, "Nucleon Axial Matrix Elements," nucl-th/9806036; J.L. Goity, R. Lewis, and M. Schvelinger, "The Goldberger-Treiman Discrepancy in 
$\mathrm{SU}(3), "$ hep-ph/9901374.

32. N. Kroll and M.A. Ruderman, Phys. Rev. 93, 233 (1954).

33. P. deBaenst, Nucl. Phys. B24, 613 (1970).

34. J.P. Burg, Ann. De Phys. (Paris) 10, 363 (1965).

35. M.J. Adamovitch et al., Sov. J. Nucl. Phys. 2, 95 (1966).

36. J. Bergstrom, private communication.

37. E.L. Goldwasser et al., Proc. XII Int. Conf. on High Energy Physics, Dubna, 1964, ed. Ya.-A Smorodinsky, Atomizdat, Moscow (1966).

38. M. Kovash, $\pi N$ Newsletter 12, 51 (1997).

39. E. Jenkins and A.V. Manohar, in Effective Theories of the Standard Model, ed. U.-G. Meißner, World Scientific, Singapore (1992).

40. Here we follow V. Bernard, N. Kaiser, J. Kambor and Ulf-G. Meißner, Nucl. Phys. B388, 315 (1992).

41. F. Low, Phys. Rev. 96, 1428 (1954); M. Gell-Mann and M.L. Goldberger, Phys. Rev. 96, 1433 (1954).

42. V. Bernard, N. Kaiser and Ulf-G. Meißner, Phys. Rev. Lett. 67, 1515 (1991); Nucl. Phys. B373, 364 (1992).

43. F.J. Federspiel et al., Phys. Rev. Lett. 67, 1511 (1991); E.L. Hallin et al., Phys. Rev. C48, 1497 (1993); A. Zeiger et al., Phys. Lett. B278, 34 (1992); B.E. MacGibben et al., Phys. Rev. C52, 2097 (1995).

44. S. Drell and A.C. Hearn, Phys. Rev. Lett. 16, 908 (1966); S. Gerasimov, Sov. J. Nucl. Phys. 2, 430 (1966).

45. V. Bernard, J. Gasser, N. Kaiser and Ulf-G. Meissner, Phys. Lett. B268, 291 (1991).

46. M. Fuchs et al., Phys. Lett. B368, 20 (1996).

47. J.C. Bergstrom et al., Phys. Rev. C53, R1052 (1996).

48. P. Argan et al., Phys. Lett. B206, 4 (1988).

49. J.F. Donoghue and E.S. Na, Phys. Rev. D56, 7073 (1997).

50. T.N. Truong, Phys. Rev. Lett. 61, 2526 (1988).

51. T. Hannah, Phys. Rev. D59, 057502 (1999).

52. R. Omnes, Nuovo Cim. 8, 1244 (1958).

53. L.S. Brown and R.L. Goble, Phys. Rev. Lett. 20, 346 (1968).

54. Chiral Dynamics: Theory and Experiment 1., ed. A. Bernstein and B.R. Holstein, Springer-Verlag, New York (1996); Chiral Dynamics: Theory and Experiment 2., ed. A. Bernstein and Th. Walcher, Springer-Verlag, New York (1998). 